\title{
Sites for priority biodiversity conservation in the Caribbean Islands Biodiversity Hotspot
}

\author{
Verónica Anadón-Irizarry ${ }^{1}$, David C. Wege ${ }^{2}$, Amy Upgren ${ }^{3}$, Richard Young ${ }^{4}$, Brian Boom ${ }^{5}$, Yolanda \\ M. León ${ }^{6}$,Yvonne Arias ${ }^{7}$, Kellee Koenig ${ }^{8}$, Alcides L. Morales ${ }^{9}$, Wayne Burke ${ }^{10}$, Amiro Pérez-Leroux \\ ${ }^{11}$, Catherine Levy ${ }^{12}$, Susan Koenig ${ }^{13}$, Lynn Gape ${ }^{14}$ \& Predensa Moore ${ }^{15}$ \\ ${ }^{1}$ BirdLife International, Rio Canas 2111, calle Colorado, Ponce, Puerto Rico 00731-1824, USA \\ ${ }^{2}$ BirdLife International, Wellbrook Court, Girton Road, Cambridge, CB3 ONA, UK \\ ${ }^{3,8}$ Conservation International, 2011 Crystal Drive, Suite 500, Arlington, VA 22202, USA \\ ${ }^{4}$ Durrell Wildlife Conservation Trust, Les Augres Manor, Trinity, Jersey, JE3 5BP Channel Islands, UK \\ ${ }^{4}$ Department of Biology and Biochemistry, University of Bath, Claverton Down, Bath, BA2 7AY, UK \\ ${ }^{5}$ The New York Botanical Garden, 2900 Southern Boulevard, Bronx, New York 10458 USA \\ ${ }^{6,7}$ Grupo Jaragua, El Vergel No.33. El Vergel Santo Domingo, D. N. República Dominicana \\ ${ }^{6}$ Instituto Tecnológico de Santo Domingo, Ave. Los Próceres Galá, Santo Domingo, República Dominicana. \\ ${ }^{9}$ Sociedad Ornitológica Puertorriqueña, Inc., 1605 Carr. 477 Quebradillas Puerto Rico 00678 \\ ${ }^{10}$ Woodbourne Shorebird Refuge, Packers, St. Patricks, Christ Church, BB17016, Barbados \\ ${ }^{11}$ BirdLife International, Juan de Dios Martínez N35-76 y Av. Portugal, Quito - Ecuador, CP 17-17-717 \\ ${ }^{12} 2$ Starlight Avenue Kingston 6 Jamaica, West Indies \\ ${ }^{13}$ Windsor Research Centre, Sherwood Content P.O., Trelawny, Jamaica, West Indies \\ ${ }^{14,15}$ Bahamas National Trust, P.O. Box N 4105, Nassau, The Bahamas \\ Email: ${ }^{1}$ veronica.anadon@birdlife.org (corresponding author), ${ }^{2}$ david.wege@birdlife.org, ${ }^{3}$ a.upgren@conservation.org, ${ }^{4}$ richard. \\ young@durrell.org, ${ }^{5}$ bboom@nybg.org, ${ }^{6}$ ymleon@yahoo.com, ymleon@intec.edu.do, ${ }^{7}$ yvonne.arias2@gmail.com, ${ }^{8}$ k.koenig@ \\ conservation.org, ${ }^{9}$ alcidesl.morales@yahoo.com, ${ }^{10}$ docinbarbados@yahoo.com, ${ }^{11}$ amiro.perez-leroux@birdlife.org, ${ }^{12}$ bluequit@ \\ gmail.com, ${ }^{13}$ windsor@cwjamaica.com, ${ }^{14}$ Igape@bnt.bs, ${ }^{15}$ pmoore@bnt.bs
}

Date of publication (online): 06 August 2012 Date of publication (print): 06 August 2012 ISSN 0974-7907 (online) | 0974-7893 (print)

\section{Manuscript details:}

Ms \# 02996

Received 08 November 2011

Final revised received 08 March 2012

Finally accepted 02 June 2012

Citation: Anadón-Irizarry, V., D.C. Wege, A Upgren, R. Young, B. Boom, Y.M. León, Y. Arias, K. Koenig, A.L. Morales, W. Burke, A. PerezLeroux, C. Levy, S. Koenig, L. Gape \& P. Moore (2012) Sites for priority biodiversity conservation in the Caribbean Islands Biodiversity Hotspot Journal of Threatened Taxa 4(8): 2806-2844.

Copyright: () Verónica Anadón-Irizarry, David C. Wege, Amy Upgren, Richard Young, Brian Boom, Yolanda M. León, Yvonne Arias, Kellee Koenig, Alcides L. Morales, Wayne Burke, Amiro Pérez-Leroux, Catherine Levy, Susan Koenig, Lynn Gape \& Predensa Moore 2012 Creative Commons Attribution 3.0 Unported License. JoTT allows unrestricted use of this article in any medium for non-profit purposes, reproduction and distribution by providing adequate credit to the authors and the source of publication.

For Author Details, Author Contribution, Acknowledgements and funding agencies/ institutions logos see end of this article.

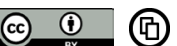

OPEN ACCESS | FREE DOWNLOAD

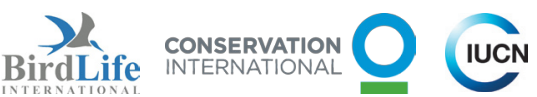 \\ BirdLife conservation $O$ INTERNATIONAL}

Abstract: The Caribbean Islands Biodiversity Hotspot is exceptionally important for global biodiversity conservation due to high levels of species endemism and threat. A total of 755 Caribbean plant and vertebrate species are considered globally threatened, making it one of the top Biodiversity Hotspots in terms of threat levels. In 2009, Key Biodiversity Areas (KBAs) were identified for the Caribbean Islands through a regionallevel analysis of accessible data and literature, followed by extensive national-level stakeholder consultation. By applying the Vulnerability criterion, a total of 284 Key Biodiversity Areas were defined and mapped as holding 409 (54\%) of the region's threatened species. Of these, 144 (or 51\%) overlapped partially or completely with protected areas. Cockpit Country, followed by Litchfield Mountain - Matheson's Run, Blue Mountains (all Jamaica) and Massif de la Hotte (Haiti) were found to support exceptionally high numbers of globally threatened taxa, with more than 40 such species at each site. Key Biodiversity Areas, building from Important Bird Areas, provide a valuable framework against which to review the adequacy of existing national protectedarea systems and also to prioritize which species and sites require the most urgent conservation attention.

Keywords: Biodiversity, BirdLife, Caribbean, hotspot, Important Bird Area, Key Biodiversity Area.

Spanish Abstract: see end of text.

The Key Biodiversity Area series documents the application of the concept and showcases the results from various parts of the world. The series is edited under the auspices of the IUCN World Commission on Protected Areas/Species Survival Commission Joint Task Force on 'Biodiversity and Protected Areas', with the editors supported by BirdLife International, Conservation International, IUCN, National Fish \& Wildlife Foundation, NatureServe, Parks Canada, and Plantlife International.

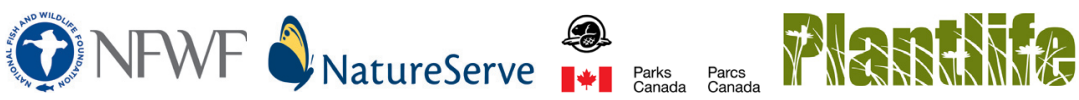




\section{INTRODUCTION}

The Caribbean Islands Biodiversity Hotspot is exceptionally important for global biodiversity conservation, due to high levels of species endemism and threat. The Caribbean is home to approximately 11,000 plants species, of which $72 \%$ are endemic to the region. The vertebrates are also characterized by extremely high levels of species endemism: $100 \%$ of 189 amphibian species, $95 \%$ of 520 reptile species, $74 \%$ of 69 mammal species and $26 \%$ of 564 species birds are unique to the Caribbean Islands. In terms of endemism at the genus level, it ranks third among the world's 34 Biodiversity Hotspots with 205 plants and 65 vertebrate genera endemic to the islands (Smith et al. 2004). Species restricted to the Caribbean Islands Biodiversity Hotspot represent $2.6 \%$ of the world's 300,000 plants species and $3.5 \%$ of the world's 27,298 vertebrate species (Wege et al. 2010).

The high level of biological diversity in the Caribbean is due to several factors. During the early Cretaceous (120 to 140 million years B.P.), a chain of volcanic islands (called Proto-Antilles) began to emerge along the eastern edge of the Caribbean Plate in the Pacific Ocean. The plate drifted eastward serving as a stepping-stone route for limited exchange of terrestrial organisms between the Nearctic and Neotropical regions. By the Eocene (58 million years B.P.), the core of the Greater Antilles achieved their present positions (Brown \& Lomolino 1998). The Lesser Antilles are the active remnants of an ancient volcanic chain, and are younger than the Greater Antilles. Several islands have particularly rugged and mountainous landscapes separated by large stretches of sea, which resulted in the isolation of populations and eventually to speciation.

The Caribbean has suffered from high levels of habitat loss since the arrival of Europeans in the 1490s. This destruction has reduced the hotspot's original estimated $229,549 \mathrm{~km}^{2}$ of natural vegetation to just $22,955 \mathrm{~km}^{2}$ (or just $10 \%$ ). The loss of native habitat combined with other threat factors, such as introduced (alien invasive) species, has resulted in severe and widespread degradation of the Caribbean's unique biodiversity. Currently, 755 plants and vertebrate species are at risk of extinction, making the region one of the biodiversity hotspots holding the most globally threatened species.

National governments and donor agencies have primarily invested in developing protected areas systems to halt biodiversity loss. However, these are rarely comprehensive in their overlap with unique species and habitats, are frequently inadequately managed, and often fail to protect important places for biodiversity. Key Biodiversity Areas (KBAs) in the Caribbean can be used as a tool for reviewing the efficacy of existing national protected-area systems. KBAs provide a site-based framework against which gaps in protected-area coverage can be identified and candidate sites for expansion. This paper has three objectives. First, to explain the identification process for Caribbean KBAs that was conducted within the context of developing the Caribbean Islands Ecosystem Profile for the Critical Ecosystem Partnership Fund. Second, to evaluate how the identified KBAs are represented in the region's existing protected-area systems, therefore highlighting key gaps in them, and to prioritize among the KBAs for conservation action. Finally, to evaluate the effectiveness of the KBA approach in guiding conservation priorities in the Caribbean Islands Hotspot.

\section{METHODS}

The Caribbean Islands Hotspot (Image 1) includes the biologically and culturally diverse islands of The Bahamas (Image 2), Greater Antilles (Puerto Rico (Image 5), Jamaica (Image 3), Cuba (Image 2) and Hispaniola [comprising the countries of the Dominican Republic and Haiti] (Image 4)), Virgin Islands, Cayman Islands, Lesser Antilles (Image 5) and the Netherlands Antilles (Image 6). It does not include Trinidad and Tobago. So defined, the hotspot represents a complex geopolitical region of 12 independent nations, and six British and three U.S. overseas territories, two French overseas départements, two French overseas collectivités, three special municipalities of the Netherlands and two constituent countries within the Kingdom of the Netherlands. The hotspot encompasses more than four million $\mathrm{km}^{2}$ of ocean (not included in the analysis) as well as c. $230,000 \mathrm{~km}^{2}$ of land area, with the four islands of Cuba, Hispaniola, Jamaica and Puerto Rico making up about $90 \%$ of this. The coastal area included in the analysis embraces territorial waters up to 12 nautical miles. The elevational range spans from over $3,000 \mathrm{~m}$ above sea level to $40 \mathrm{~m}$ below sea level and encompasses a diverse array of habitats and ecosystems. 


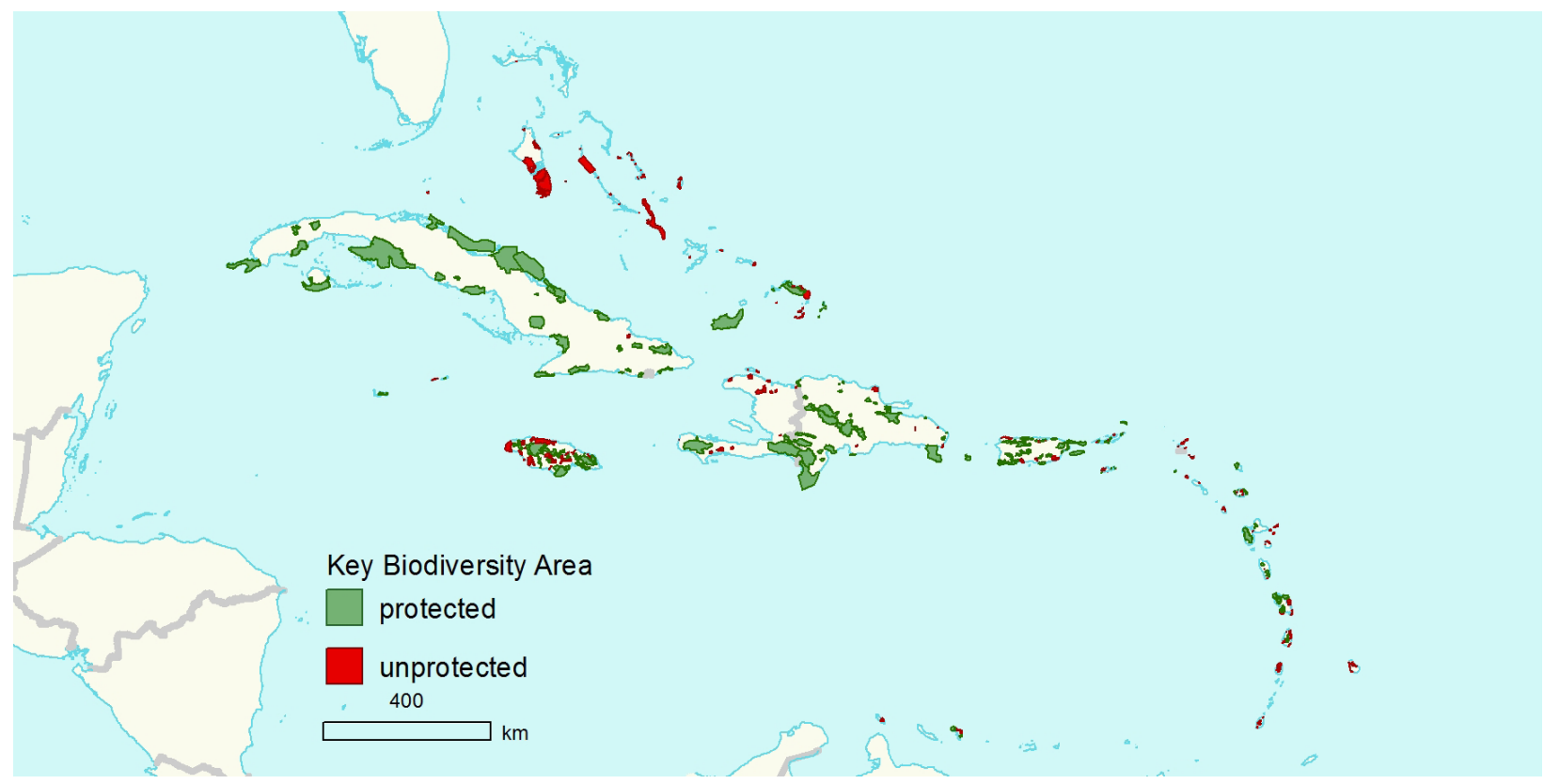

Image 1. Partially or completely protected (in green) and unprotected (in red) Key Biodiversity Areas [2010] in the Caribbean Islands Biodiversity Hotspot.

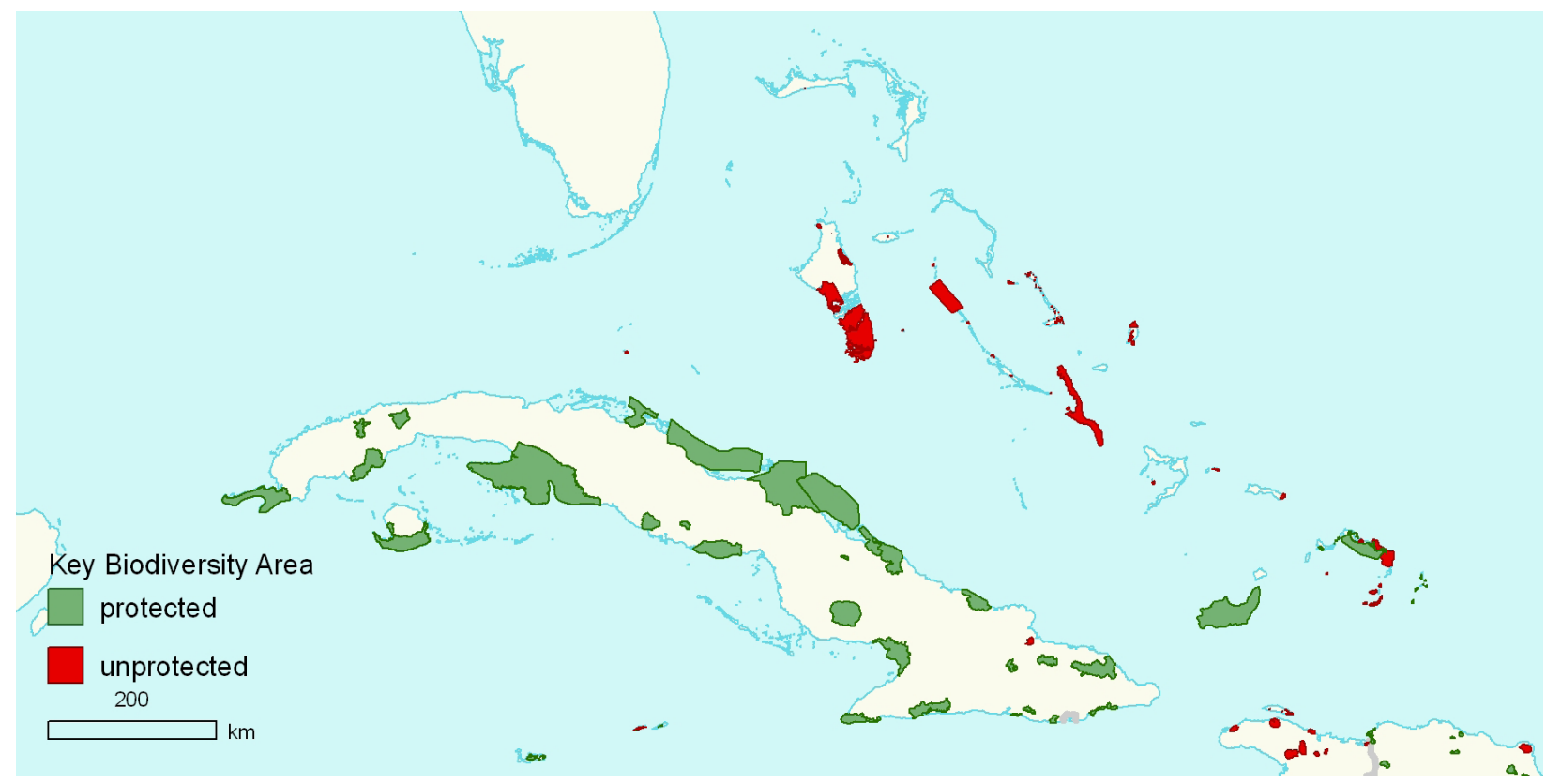

Image 2. Partially or completely protected (in green) and unprotected (in red) Key Biodiversity Areas [2010] in the Bahamas and Cuba.

The Caribbean KBAs were identified as an integral part of developing the Caribbean Islands Hotspot Ecosystem Profile for the Critical Ecosystem Partnership Fund during 2009 (Wege et al. 2010). For the Caribbean Islands Hotspot, only the Vulnerability criterion was applied to select KBAs, with the confirmed presence of globally threatened species triggering the definition of a KBA. The Irreplaceability criterion was not applied due to lack of quantitative data for other taxa in the hotspot. This criterion is currently only used to define KBAs for birds, as this is the only group for which the concept of restricted-range species has been quantitatively defined: species with global breeding ranges of less than $50,000 \mathrm{~km}^{2}$ (Stattersfield et al. 


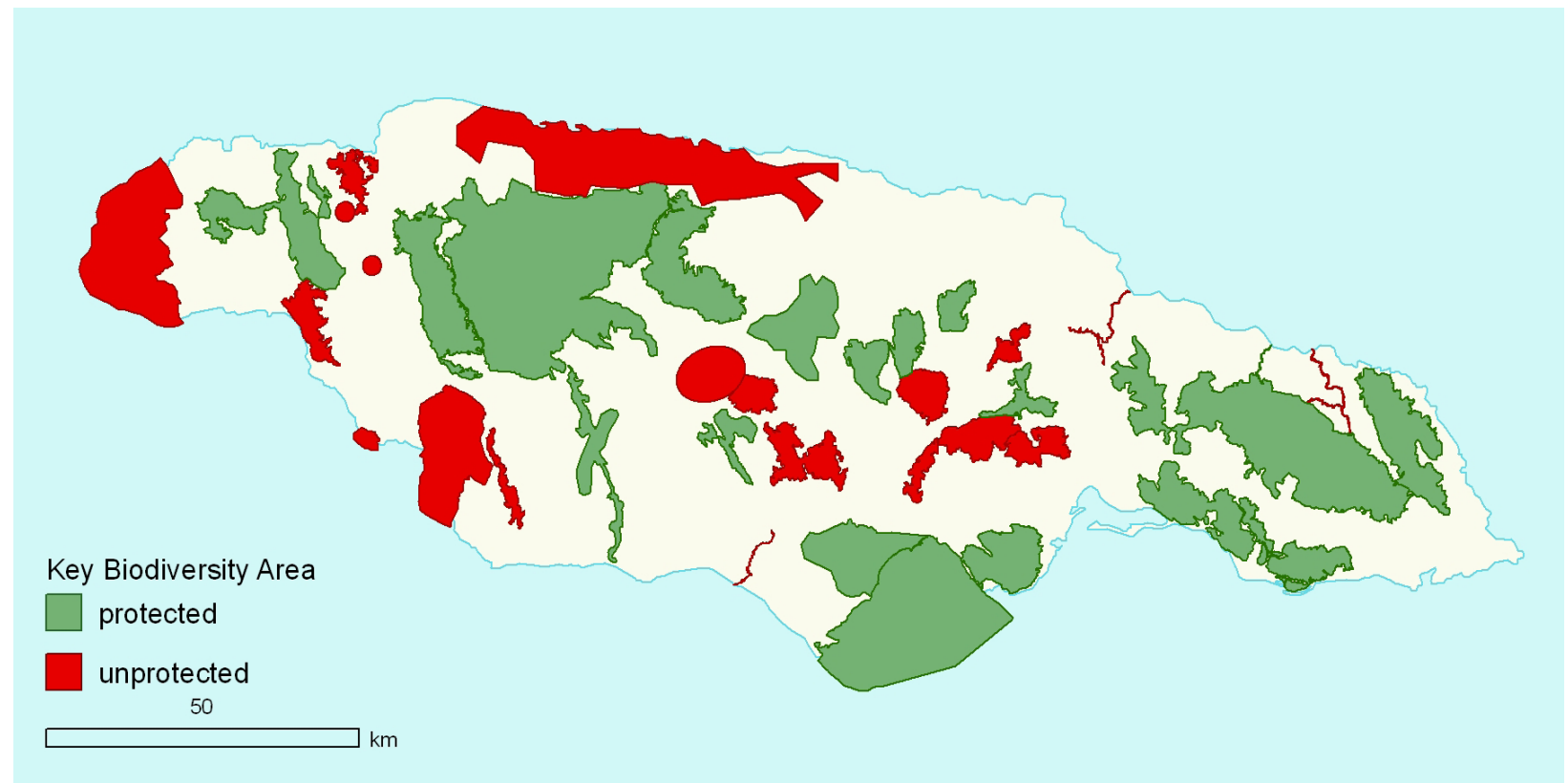

Image 3. Partially or completely protected (in green) and unprotected (in red) Key Biodiversity Areas [2010] in Jamaica.

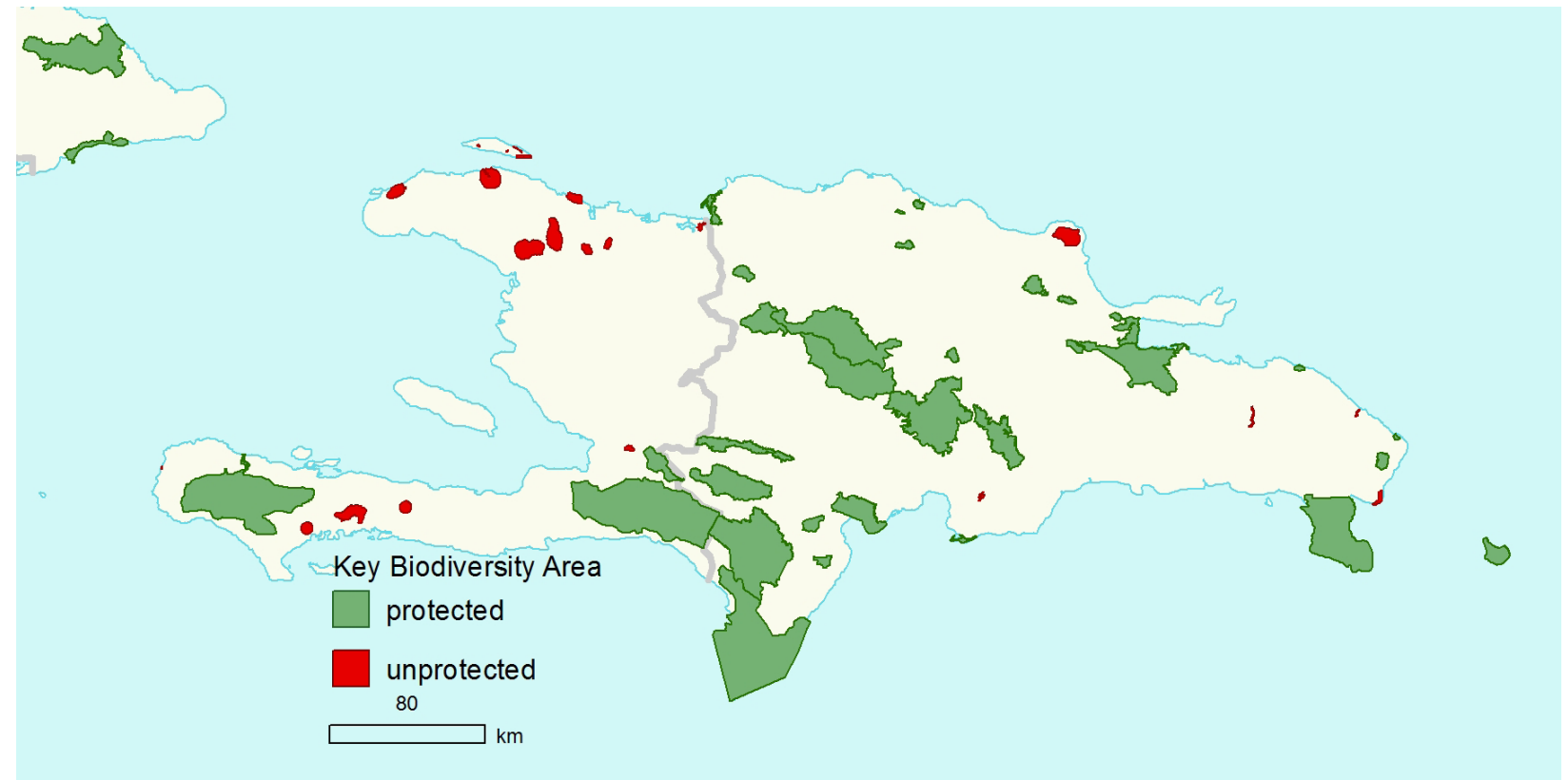

Image 4. Partially or completely protected (in green) and unprotected (in red) Key Biodiversity Areas [2010] in Hispaniola.

1998). However, to prevent a bias toward site priorities for birds, KBAs in the Caribbean Islands Hotspot are identified only based on the Vulnerability criterion.

The KBA identification process comprised a deskbased, regional-level analysis of accessible data and literature, followed by national-level stakeholder consultations coordinated by BirdLife International, in collaboration with the Durrell Wildlife Conservation Trust, the University of Bath and the New York
Botanical Garden, and with technical support from Conservation International. Important Bird Areas (IBAs), documented by BirdLife partners and collaborating organizations in 2008 (BirdLife International 2008), were used as a scientifically robust starting point for KBA identification.

This foundation of IBAs was supplemented by applying the Vulnerability criterion to non-avian taxa to define terrestrial KBAs based on the occurrence of 


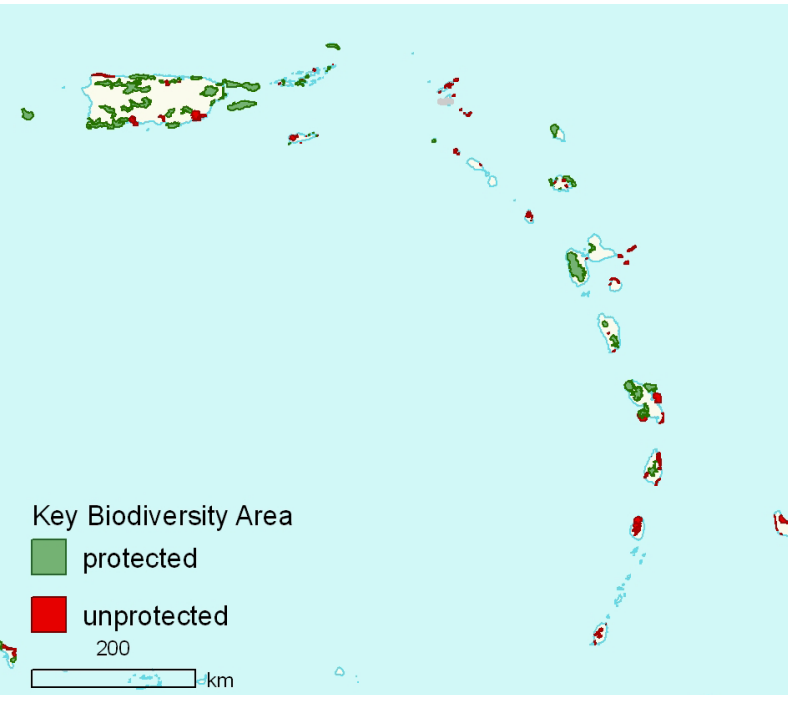

Image 5. Partially or completely protected (in green) and unprotected (in red) Key Biodiversity Areas [2010] in Puerto Rico and the Lesser Antilles.

globally threatened species as categorized on the 2008 IUCN Red List (a global, standardized assessment of species threat status). The taxonomic groups used to define KBAs were land-based mammals, birds (through the IBA process previously mentioned), amphibians, reptiles and plants. Sea turtle nesting beaches were included in the analysis where more than 100 crawls annually had been recorded (Dow et al. 2007). KBAs were delineated using a geographic information system (GIS) and by taking into consideration the distribution of available habitat for the globally threatened species, and also land/ protected area management units. The degree of protection of the KBAs was analyzed against the 2010 World Database on Protected Areas (WDPA).

National profile coordinators in The Bahamas (Bahamas National Trust), Dominican Republic (Grupo Jaragua), Haiti (Société Audubon Haïti), Jamaica (BirdLife), Lesser Antilles (BirdLife in Barbados) and Puerto Rico (Sociedad Ornitológica Puertorriqueña) facilitated a review of priorities within their own countries. In Cuba, it was not possible to conduct the analysis for taxonomic groups other than birds. National workshops were held in Dominican Republic, Haiti and Jamaica during June 2009, with a region-wide workshop held in July 2009 on Antigua as a formal part of the $17^{\text {th }}$ Regional Meeting of the Society for the Conservation and Study of Caribbean Birds.

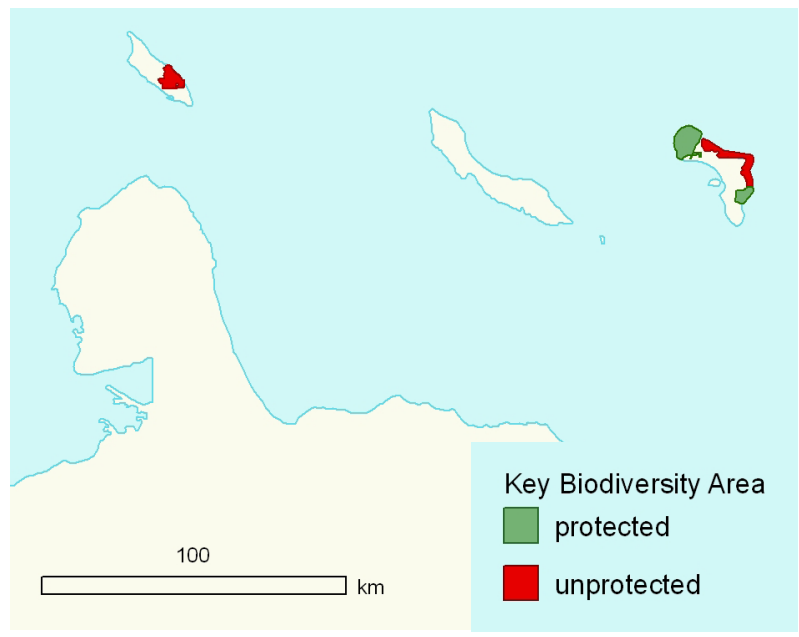

Image 6. Partially or completely protected (in green) and unprotected (in red) Key Biodiversity Areas [2010] in Aruba and Bonaire.

\section{RESULTS}

A total of 284 Key Biodiversity Areas were defined for all the countries and territories contained within the Caribbean Islands Biodiversity Hotspot (Table 1). The 284 KBAs in the Caribbean Islands Hotspot cover just over $50,000 \mathrm{~km}^{2}$, or roughly $22 \%$ of the terrestrial and coastal portion of the hotspot. Half of the KBAs are either fully or partially protected according to the 2010 WDPA (Images 1-6).

In total, 409 globally threatened plants and vertebrate species triggered the Vulnerability criterion to define KBAs (hereafter referred as trigger species). No data were available for the other 346 globally threatened species, most of which were plants, at the time of this analysis. Plants had the highest number of globally threatened species, while for vertebrate species groups, amphibians and then birds were the most abundant groups (Table 2). However, globally threatened birds were responsible for defining the largest number of KBAs, followed by amphibians and plants (Table 3). The majority of KBAs were defined by the presence of multiple globally threatened trigger species, with almost half of all KBAs (141 or 49.64\%) supporting two to 10 trigger species and 28 (or $9.86 \%$ ) by 11 to 44 species. Cockpit Country in Jamaica supports an incredible 60 globally threatened species, followed by Litchfield Mountain-Matheson's Run, Blue Mountains (both with 44) and Massif de la Hotte in 
Table 1. Geographical summary of the countries included in the Key Biodiversity Areas analysis for the Caribbean Islands Biodiversity Hotspot.

\begin{tabular}{|c|c|c|c|c|c|c|}
\hline Country/ territory & Area (ha) & $\begin{array}{l}\text { No. } \\
\text { KBAs }\end{array}$ & $\begin{array}{l}\text { Area of } \\
\text { KBAs (ha) }\end{array}$ & $\begin{array}{l}\% \text { country } \\
\text { in KBAs* }\end{array}$ & $\begin{array}{l}\text { No. of KBAs protected/ } \\
\text { partially protected }\end{array}$ & $\begin{array}{l}\% \text { of KBAs protected/ } \\
\text { partially protected }\end{array}$ \\
\hline Anguilla & 9,800 & 6 & 3,885 & 40 & 0 & 0 \\
\hline Antigua and Barbuda & 44,100 & 10 & 18,221 & 41 & 4 & 40 \\
\hline Aruba & 19,300 & 1 & 3,801 & 20 & 0 & 0 \\
\hline Bahamas & $1,394,000$ & 26 & 544,158 & 39 & 2 & 8 \\
\hline Barbados & 43,100 & 5 & 5,776 & 13 & 0 & 0 \\
\hline British Virgin Islands & 15,300 & 6 & 6,319 & 41 & 3 & 50 \\
\hline Cayman Islands & 26,200 & 8 & 6,515 & 25 & 6 & 75 \\
\hline Cuba & $10,988,600$ & 28 & $2,310,384$ & 21 & 27 & 96 \\
\hline Dominica & 75,400 & 4 & 10,948 & 15 & 2 & 50 \\
\hline Dominican Republic & $4,873,000$ & 32 & 868,314 & 18 & 28 & 88 \\
\hline Grenada & 34,400 & 9 & 3,321 & 10 & 0 & 0 \\
\hline Guadeloupe & 171,300 & 8 & 48,563 & 28 & 2 & 25 \\
\hline Haiti & $2,775,000$ & 17 & 360,314 & 13 & 3 & 18 \\
\hline Jamaica & $1,082,900$ & 38 & 444,903 & 41 & 21 & 55 \\
\hline Martinique & 110,000 & 8 & 54,522 & 50 & 8 & 100 \\
\hline Montserrat & 10,200 & 3 & 1,652 & 16 & 0 & 0 \\
\hline $\begin{array}{l}\text { Netherlands Antilles } \\
\text { (Bonaire, Curaçao, } \\
\text { Saba, St Eustatius, St } \\
\text { Maarten) }\end{array}$ & 79,900 & 7 & 18,372 & 23 & 4 & 57 \\
\hline Puerto Rico & 887,000 & 27 & 231,524 & 26 & 24 & 89 \\
\hline St Barthélemy & 2,500 & 4 & 1,452 & 58 & 0 & 0 \\
\hline St Kitts and Nevis & 26,100 & 1 & 16 & 0.06 & 0 & 0 \\
\hline St Lucia & 61,600 & 6 & 15,537 & 25 & 3 & 50 \\
\hline St Martin & 5,600 & 1 & 663 & 12 & 0 & 0 \\
\hline $\begin{array}{l}\text { St Vincent and the } \\
\text { Grenadines }\end{array}$ & 38,900 & 7 & 13,221 & 34 & 0 & 0 \\
\hline $\begin{array}{l}\text { Turks and Caicos } \\
\text { Islands }\end{array}$ & 50,000 & 10 & 106,001 & 212 & 0 & 0 \\
\hline US Virgin Islands & 35,300 & 12 & 8,435 & 24 & 7 & 58 \\
\hline Total & $22,859,500$ & 284 & $5,086,816$ & 22 & 144 & 51 \\
\hline
\end{tabular}

The percent coverage by KBAs can be over $100 \%$ as some KBAs include both land and water area, while the total area for each country is calculated based solely on land area. Cuban key biodiversity areas comprise only sites (IBAs) identified as important for globally threatened birds.

Table 2. Total number of species triggering the Vulnerability criterion for each higher taxonomic group

\begin{tabular}{|l|c|c|c|c|}
\hline & \multicolumn{3}{|c|}{ Vulnerability } & \\
\hline $\begin{array}{l}\text { Taxonomic } \\
\text { Group }\end{array}$ & $\begin{array}{c}\text { Critically } \\
\text { Endangered }\end{array}$ & Endangered & Vulnerable & Total \\
\hline Amphibians & 47 & 36 & 11 & 94 \\
\hline Birds & 11 & 16 & 21 & 48 \\
\hline Mammals & 1 & 2 & 11 & 14 \\
\hline Plants & 52 & 58 & 109 & 219 \\
\hline Reptiles & 16 & 9 & 9 & 34 \\
\hline Total & 127 & 121 & 161 & 409 \\
\hline
\end{tabular}

Table 3. Total number of KBAs triggered by the Vulnerability criterion for each higher taxonomic group

\begin{tabular}{|l|c|c|c|}
\hline & \multicolumn{3}{|c|}{ Vulnerability } \\
\hline $\begin{array}{l}\text { Taxonomic } \\
\text { Group }\end{array}$ & $\begin{array}{c}\text { Critically } \\
\text { Endangered }\end{array}$ & Endangered & Vulnerable \\
\hline Amphibians & 42 & 75 & 53 \\
\hline Birds & 19 & 61 & 121 \\
\hline Mammals & 2 & 15 & 49 \\
\hline Plants & 33 & 71 & 58 \\
\hline Reptiles & 52 & 25 & 61 \\
\hline
\end{tabular}


Table 4. Total frequency distributions of the number of trigger species per KBA, and the number of KBAs per trigger species in the Caribbean Islands

\begin{tabular}{|c|c|c|}
\hline $\mathrm{n}$ & $\begin{array}{c}\text { \# KBAs holding } \mathrm{n} \text { trigger } \\
\text { species }\end{array}$ & $\begin{array}{l}\text { \# trigger species occurring } \\
\text { in } \mathrm{n} \text { KBAs }\end{array}$ \\
\hline 1 & 114 & 173 \\
\hline 2 & 37 & 80 \\
\hline 3 & 26 & 43 \\
\hline 4 & 23 & 27 \\
\hline 5 & 14 & 19 \\
\hline 6 & 12 & 9 \\
\hline 7 & 10 & 7 \\
\hline 8 & 8 & 9 \\
\hline 9 & 5 & 9 \\
\hline 10 & 6 & 6 \\
\hline 11 & 2 & 7 \\
\hline 12 & 3 & 4 \\
\hline 13 & 1 & 2 \\
\hline 14 & 0 & 2 \\
\hline 15 & 3 & 0 \\
\hline 16 & 2 & 2 \\
\hline 17 & 1 & 3 \\
\hline 18 & 2 & 2 \\
\hline 19 & 0 & 0 \\
\hline 20 & 1 & 1 \\
\hline 21 & 0 & 0 \\
\hline 22 & 0 & 2 \\
\hline 23 & 1 & 0 \\
\hline 24 & 1 & 1 \\
\hline 25 & 1 & 0 \\
\hline 26 & 3 & 0 \\
\hline 27 & 2 & 0 \\
\hline 28 & 0 & 0 \\
\hline 29 & 0 & 0 \\
\hline 30 & 0 & 0 \\
\hline 31 & 0 & 0 \\
\hline 32 & 0 & 0 \\
\hline 33 & 1 & 0 \\
\hline 34 & 1 & 0 \\
\hline 35 & 0 & 0 \\
\hline 36 & 0 & 0 \\
\hline 37 & 0 & 0 \\
\hline 38 & 0 & 0 \\
\hline 39 & 0 & 0 \\
\hline 40 & 0 & 0 \\
\hline 41 & 0 & 0 \\
\hline 42 & 1 & 0 \\
\hline 43 & 0 & 0 \\
\hline 44 & 2 & 0 \\
\hline 45 & 0 & 0 \\
\hline 46 & 0 & 0 \\
\hline 47 & 0 & 0 \\
\hline 48 & 0 & 0 \\
\hline 49 & 0 & 0 \\
\hline 50 & 0 & 0 \\
\hline 51 & 0 & 0 \\
\hline 52 & 0 & 0 \\
\hline 53 & 0 & 0 \\
\hline 54 & 0 & 1 \\
\hline 55 & 0 & 0 \\
\hline 56 & 0 & 0 \\
\hline 57 & 0 & 0 \\
\hline 58 & 0 & 0 \\
\hline 59 & 0 & 0 \\
\hline 60 & 1 & 0 \\
\hline Total & 284 & 409 \\
\hline
\end{tabular}

Haiti (with 42). On the other hand, 114 (or 40\%) KBAs were identified for a single trigger species (Table 4).

A total of 173 (or $42.29 \%$ ) of the trigger species have distributions confined to just one KBA (see Table 5). However, $25 \mathrm{KBAs}$ hold populations of more than one of these "single-site" species with Cockpit Country, Blue Mountains and Massif de la Hotte each supporting populations of more than 20 such species. An important result of the KBA process was the identification of $56 \mathrm{KBAs}$ which contained the only record of a globally threatened species in the Caribbean Islands Biodiversity Hotspot. No fewer than 19 of these KBAs were identified in 2010 as global Alliance for Zero Extinction (AZE) sites (Tables 4 and 5) - defined by the presence of Critically Endangered or Endangered species confined to just a single site. One hundred and sixty nine (169 or $41.32 \%$ ) of the trigger species occur only in two to five KBAs and the other 67 (16.38\%) trigger species occur in more than six KBAs (Table 4).

\section{DISCUSSION}

The countries with the greatest numbers of Key Biodiversity Areas are the large islands of the Greater Antilles and multi-island countries such as The Bahamas. This is to be expected as the principles of island biogeography dictate that the larger (and older) the island, the greater the species diversity. Higher species diversity on each of the Greater Antilles, combined with greater ecosystem, habitat and altitudinal diversity, has led to larger numbers of endemic species and consequently higher numbers of globally threatened taxa. Small islands in archipelagos such as The Bahamas often result in taxonomic isolation and the presence here of globally threatened species occupying very small ranges (often confined to a single island) has in turn led to the definition of relatively large numbers of KBAs. In Cuba, the KBAs included only IBAs as it was not possible to incorporate the results of analyses of other taxonomic groups or consultations with experts for the definition of other sites.

The amount and quality of available data on the distribution of globally threatened species among Caribbean KBAs vary between taxonomic groups but the sites identified will almost certainly be important for other groups for which data are not currently available. However, there are likely to be additional 
sites holding globally threatened species that have not been identified during this process. This is because reptiles, plants (especially cacti and orchids) and bats have not been systematically assessed against Red List criteria. Neither have freshwater fish (of which there are numerous endemics in the region), such that just five have so far been categorized as globally threatened, and no KBAs were defined for this group.

In addition to the occurrence of globally threatened species (the Vulnerability criterion), KBAs can also be defined on the basis of the presence of restricted-range species (the Irreplaceability criterion): their inclusion as a next step may result in a better coverage of the poorly represented taxonomic groups mentioned above.

Table 5. Summary data for species triggering the Vulnerability criterion at only one KBA in the Caribbean Islands Biodiversity Hotspot

\begin{tabular}{|c|c|c|c|}
\hline Country/territory & Key Biodiversity Area & Species & $\begin{array}{l}\text { IUCN Red List } \\
\text { category }\end{array}$ \\
\hline Antigua and Barbuda & Offshore Islands & Alsophis antiguae & $\mathrm{CR}$ \\
\hline Aruba & Arikok National Park & Crotalus unicolor & $C R$ \\
\hline Bahamas & Cay Sal & Caretta caretta & EN \\
\hline Barbados & Scotland District & Liophis perfuscus & EN \\
\hline British Virgin Islands & Anegada Island & Acacia anegadensis & CR \\
\hline British Virgin Islands & Anegada Island & Metastelma anegadense & $\mathrm{CR}$ \\
\hline British Virgin Islands & Virgin Gorda & Calyptranthes kiaerskovii & VU \\
\hline Cuba & Alejandro de Humboldt * & Campephilus principalis & $\mathrm{CR}$ \\
\hline Cuba & Alejandro de Humboldt * & Chondrohierax wilsonii & $\mathrm{CR}$ \\
\hline Cuba & Ciénaga de Zapata * & Ferminia cerverai & EN \\
\hline Cuba & Ciénaga de Zapata * & Cyanolimnas cerverai & $\mathrm{CR}$ \\
\hline Dominica & Morne Trois Pitons National Park & Phycolepidozia exigua & $\mathrm{CR}$ \\
\hline Dominican Republic & Bahoruco Oriental & Antirhea radiata & VU \\
\hline Dominican Republic & Loma La Humeadora & Ateleia gummifera & EN \\
\hline Dominican Republic & Loma La Humeadora & Celestus anelpistus & $\mathrm{CR}$ \\
\hline Dominican Republic & Loma La Humeadora & Pimenta cainitoides & VU \\
\hline Dominican Republic & Los Haitises * & Abarema abbottii & $\mathrm{VU}$ \\
\hline Dominican Republic & Los Haitises * & Buteo ridgwayi & CR \\
\hline Dominican Republic & Los Haitises * & Sideroxylon dominicanum & VU \\
\hline Dominican Republic & Parque Nacional Jaragua & Pseudophoenix ekmanii & CR \\
\hline Dominican Republic & Parque Nacional Jaragua & Pimenta haitiensis & VU \\
\hline Dominican Republic & Sierra de Neyba & Eleutherodactylus parabates & $\mathrm{CR}$ \\
\hline Dominican Republic & Valle Nuevo & Cleyera vaccinioides & $\mathrm{VU}$ \\
\hline Guadeloupe & Massif forestier de l'île de Basse-Terre * & Eleutherodactylus barlagnei & EN \\
\hline Guadeloupe & Massif forestier de l'île de Basse-Terre * & Eleutherodactylus pinchoni & EN \\
\hline Guadeloupe & Massif forestier de l'île de Basse-Terre * & Myotis dominicensis & $\mathrm{VU}$ \\
\hline Haiti & Dame-Marie * & Eleutherodactylus caribe & $\mathrm{CR}$ \\
\hline Haiti & Ile de la Tortue Forest & Eleutherodactylus warreni & $\mathrm{CR}$ \\
\hline Haiti & Massif de la Hotte * & Calyptranthes ekmanii & $\mathrm{VU}$ \\
\hline Haiti & Massif de la Hotte * & Catalpa brevipes & VU \\
\hline Haiti & Massif de la Hotte * & Eleutherodactylus amadeus & $\mathrm{CR}$ \\
\hline Haiti & Massif de la Hotte * & Eleutherodactylus apostates & $\mathrm{CR}$ \\
\hline Haiti & Massif de la Hotte * & Eleutherodactylus bakeri & $\mathrm{CR}$ \\
\hline Haiti & Massif de la Hotte * & Eleutherodactylus brevirostris & $\mathrm{CR}$ \\
\hline Haiti & Massif de la Hotte * & Eleutherodactylus chlorophenax & $\mathrm{CR}$ \\
\hline Haiti & Massif de la Hotte * & Eleutherodactylus corona & $\mathrm{CR}$ \\
\hline Haiti & Massif de la Hotte * & Eleutherodactylus counouspeus & EN \\
\hline Haiti & Massif de la Hotte * & Eleutherodactylus dolomedes & $\mathrm{CR}$ \\
\hline Haiti & Massif de la Hotte * & Eleutherodactylus eunaster & CR \\
\hline Haiti & Massif de la Hotte * & Eleutherodactylus glandulifer & $\mathrm{CR}$ \\
\hline Haiti & Massif de la Hotte * & Eleutherodactylus lamprotes & $\mathrm{CR}$ \\
\hline Haiti & Massif de la Hotte * & Eleutherodactylus parapelates & $\mathrm{CR}$ \\
\hline Haiti & Massif de la Hotte * & Eleutherodactylus sciagraphus & $\mathrm{CR}$ \\
\hline Haiti & Massif de la Hotte * & Eleutherodactylus thorectes & $\mathrm{CR}$ \\
\hline
\end{tabular}




\begin{tabular}{|c|c|c|c|}
\hline Country/territory & Key Biodiversity Area & Species & $\begin{array}{l}\text { IUCN Red List } \\
\text { category }\end{array}$ \\
\hline Haiti & Massif de la Hotte * & Eleutherodactylus ventrilineatus & $\mathrm{CR}$ \\
\hline Haiti & Massif de la Hotte * & Nectandra caudatoacuminata & $\mathrm{CR}$ \\
\hline Haiti & Massif de la Hotte * & Nectandra pulchra & $\mathrm{CR}$ \\
\hline Haiti & Massif de la Hotte * & Micropholis polita & VU \\
\hline Haiti & Massif de la Selle * & Eleutherodactylus darlingtoni & $\mathrm{CR}$ \\
\hline Haiti & Massif de la Selle * & Eleutherodactylus glanduliferoides & $\mathrm{CR}$ \\
\hline Haiti & Plaisance & Celestus warreni & CR \\
\hline Haiti & Presqu'ile du Nord-Ouest I * & Eleutherodactylus grahami & EN \\
\hline Haiti & Presqu'ile du Nord-Ouest I * & Eleutherodactylus Iucioi & $\mathrm{CR}$ \\
\hline Haiti & Presqu'ile du Nord-Ouest II * & Eleutherodactylus rhodesi & $\mathrm{CR}$ \\
\hline Jamaica & Black River Great Morass & Eugenia brownei & VU \\
\hline Jamaica & Blue Mountains * & Annona praetermissa & VU \\
\hline Jamaica & Blue Mountains * & Ardisia brittonii & EN \\
\hline Jamaica & Blue Mountains * & Bunchosia jamaicensis & VU \\
\hline Jamaica & Blue Mountains * & Eleutherodactylus alticola & $\mathrm{CR}$ \\
\hline Jamaica & Blue Mountains * & Eleutherodactylus nubicola & EN \\
\hline Jamaica & Blue Mountains * & Eugenia brachythrix & VU \\
\hline Jamaica & Blue Mountains * & Eugenia nicholsii & EN \\
\hline Jamaica & Blue Mountains * & Hernandia catalpifolia & VU \\
\hline Jamaica & Blue Mountains * & Hyeronima jamaicensis & VU \\
\hline Jamaica & Blue Mountains * & Maytenus harrisii & $\mathrm{CR}$ \\
\hline Jamaica & Blue Mountains * & Psychotria foetens & VU \\
\hline Jamaica & Blue Mountains * & Rondeletia brachyphylla & EN \\
\hline Jamaica & Blue Mountains * & Rondeletia hirsuta & VU \\
\hline Jamaica & Blue Mountains * & Rondeletia hirta & VU \\
\hline Jamaica & Blue Mountains * & Sebastiania alpina & VU \\
\hline Jamaica & Blue Mountains * & Verbesina rupestris & VU \\
\hline Jamaica & Blue Mountains * & Wallenia fawcettii & $\mathrm{VU}$ \\
\hline Jamaica & Blue Mountains * & Zanthoxylum hartii & VU \\
\hline Jamaica & Blue Mountains * & Brunfelsia jamaicensis & VU \\
\hline Jamaica & Blue Mountains * & Miconia nubicola & EN \\
\hline Jamaica & Blue Mountains * & Nowellia wrightii & VU \\
\hline Jamaica & Bluefields & Eugenia abbreviata & EN \\
\hline Jamaica & Bull Bay & Brunfelsia membranacea & VU \\
\hline Jamaica & Bull Bay & Phialanthus jamaicensis & EN \\
\hline Jamaica & Bull Bay & Portlandia albiflora & $\mathrm{CR}$ \\
\hline Jamaica & Bull Bay & Tetrasiphon jamaicensis & EN \\
\hline Jamaica & Catadupa * & Ouratea elegans & $\mathrm{CR}$ \\
\hline Jamaica & Catadupa * & Phyllanthus cauliflorus & VU \\
\hline Jamaica & Cockpit Country * & Bursera aromatica & VU \\
\hline Jamaica & Cockpit Country * & Buxus arborea & VU \\
\hline Jamaica & Cockpit Country * & Calliandra comosa & VU \\
\hline Jamaica & Cockpit Country * & Calyptranthes nodosa & VU \\
\hline Jamaica & Cockpit Country * & Cassipourea brittoniana & EN \\
\hline Jamaica & Cockpit Country * & Cassipourea subcordata & $\mathrm{CR}$ \\
\hline Jamaica & Cockpit Country * & Cordia harrisii & VU \\
\hline Jamaica & Cockpit Country * & Eleutherodactylus sisyphodemus & $\mathrm{CR}$ \\
\hline Jamaica & Cockpit Country * & Eugenia laurae & EN \\
\hline Jamaica & Cockpit Country * & Eugenia sachetae & EN \\
\hline Jamaica & Cockpit Country * & Exostema orbiculatum & $\mathrm{CR}$ \\
\hline Jamaica & Cockpit Country * & Hamelia papillosa & VU \\
\hline Jamaica & Cockpit Country * & Manilkara excisa & EN \\
\hline Jamaica & Cockpit Country * & Mitranthes macrophylla & CR \\
\hline Jamaica & Cockpit Country * & Pimenta richardii & EN \\
\hline Jamaica & Cockpit Country * & Psychotria plicata & VU \\
\hline Jamaica & Cockpit Country * & Psychotria siphonophora & EN \\
\hline
\end{tabular}




\begin{tabular}{|c|c|c|c|}
\hline Country/territory & Key Biodiversity Area & Species & $\begin{array}{l}\text { IUCN Red List } \\
\text { category }\end{array}$ \\
\hline Jamaica & Cockpit Country * & Rondeletia amplexicaulis & EN \\
\hline Jamaica & Cockpit Country * & Sophora saxicola & EN \\
\hline Jamaica & Cockpit Country * & Spathelia coccinea & $\mathrm{CR}$ \\
\hline Jamaica & Cockpit Country * & Strempeliopsis arborea & VU \\
\hline Jamaica & Cockpit Country * & Xylosma proctorii & VU \\
\hline Jamaica & Cockpit Country * & Phyllanthus axillaris & EN \\
\hline Jamaica & Dolphin Head & Calyptranthes discolor & EN \\
\hline Jamaica & Dolphin Head & Cassipourea subsessilis & $\mathrm{CR}$ \\
\hline Jamaica & Dolphin Head & Comocladia parvifoliola & $\mathrm{CR}$ \\
\hline Jamaica & Dolphin Head & Dendropanax cordifolius & $\mathrm{CR}$ \\
\hline Jamaica & Dolphin Head & Eugenia polypora & $\mathrm{CR}$ \\
\hline Jamaica & Dolphin Head & Lasiocroton fawcettii & VU \\
\hline Jamaica & Dolphin Head & Ormosia jamaicensis & EN \\
\hline Jamaica & Dolphin Head & Psychotria hanoverensis & $\mathrm{CR}$ \\
\hline Jamaica & Dolphin Head & Rondeletia cincta & $\mathrm{CR}$ \\
\hline Jamaica & Dolphin Head & Sebastiania fasciculata & EN \\
\hline Jamaica & Dolphin Head & Tabernaemontana ochroleuca & VU \\
\hline Jamaica & Dolphin Head & Tabernaemontana ovalifolia & EN \\
\hline Jamaica & Don Figuerero Mountains & Coccoloba proctorii & EN \\
\hline Jamaica & Hellshire Hills * & Cyclura collei & $\mathrm{CR}$ \\
\hline Jamaica & Hellshire Hills * & Phialanthus revolutus & EN \\
\hline Jamaica & Hellshire Hills * & Siphonorhis americana & $\mathrm{CR}$ \\
\hline Jamaica & John Crow Mountains * & Clusia portlandiana & VU \\
\hline Jamaica & John Crow Mountains * & Dendropanax blakeanus & $\mathrm{VU}$ \\
\hline Jamaica & John Crow Mountains * & Myrcia calcicola & VU \\
\hline Jamaica & John Crow Mountains * & Phyllanthus eximius & VU \\
\hline Jamaica & John Crow Mountains * & Psychotria bryonicola & $\mathrm{CR}$ \\
\hline Jamaica & John Crow Mountains * & Psychotria clusioides & EN \\
\hline Jamaica & John Crow Mountains * & Pterodroma caribbaea & $\mathrm{CR}$ \\
\hline Jamaica & John Crow Mountains * & Rondeletia portlandensis & VU \\
\hline Jamaica & John Crow Mountains * & Sideroxylon bullatum & VU \\
\hline Jamaica & John Crow Mountains * & Ternstroemia howardiana & VU \\
\hline Jamaica & John Crow Mountains * & Wallenia sylvestris & VU \\
\hline Jamaica & John Crow Mountains * & Weinmannia portlandiana & VU \\
\hline Jamaica & Litchfield Mountain - Matheson's Run & Calyptranthes capitata & $\mathrm{VU}$ \\
\hline Jamaica & Litchfield Mountain - Matheson's Run & Eugenia aboukirensis & $\mathrm{CR}$ \\
\hline Jamaica & Litchfield Mountain - Matheson's Run & Ocotea staminoides & EN \\
\hline Jamaica & Mount Diablo & Eugenia acutisepala & EN \\
\hline Jamaica & Mount Diablo & Gymnanthes glandulosa & VU \\
\hline Jamaica & Mount Diablo & Ilex florifera & $\mathrm{VU}$ \\
\hline Jamaica & Mount Diablo & Ilex subtriflora & $\mathrm{CR}$ \\
\hline Jamaica & Mount Diablo & Malpighia cauliflora & EN \\
\hline Jamaica & Negril & Zanthoxylum negrilense & EN \\
\hline Jamaica & Portland Ridge and Bight * & Eleutherodactylus cavernicola & $\mathrm{CR}$ \\
\hline Jamaica & Santa Cruz Mountains & Rhamnidium dictyophyllum & EN \\
\hline Martinique & Forêts du Nord et de la Montagne Pelée & Allobates chalcopis & VU \\
\hline Martinique & Pitons du Carbet & Freziera cordata & VU \\
\hline Martinique & Pitons du Carbet & Inga martinicensis & VU \\
\hline Martinique & Pitons du Carbet & Schefflera urbaniana & VU \\
\hline Martinique & Rocher du Diamant & Liophis cursor & $\mathrm{CR}$ \\
\hline Montserrat & Centre Hills * & Diploglossus montisserrati & $\mathrm{CR}$ \\
\hline Puerto Rico & Carite & Eleutherodactylus jasperi & $\mathrm{CR}$ \\
\hline Puerto Rico & Cordillera Central & Callicarpa ampla & CR \\
\hline Puerto Rico & Cordillera Central & Ilex cookii & $\mathrm{CR}$ \\
\hline Puerto Rico & Cordillera Central & Marlierea sintenisii & $\mathrm{VU}$ \\
\hline Puerto Rico & Culebra & Anolis roosevelti & $\mathrm{CR}$ \\
\hline
\end{tabular}




\begin{tabular}{|c|c|c|c|}
\hline Country/territory & Key Biodiversity Area & Species & $\begin{array}{l}\text { IUCN Red List } \\
\text { category }\end{array}$ \\
\hline Puerto Rico & El Yunque * & Eleutherodactylus unicolor & VU \\
\hline Puerto Rico & El Yunque * & Styrax portoricensis & $\mathrm{CR}$ \\
\hline Puerto Rico & El Yunque * & Ternstroemia subsessilis & $\mathrm{CR}$ \\
\hline Puerto Rico & Karso del Norte & Henriettea membranifolia & CR \\
\hline Puerto Rico & Karso del Norte & Myrcia paganii & $\mathrm{CR}$ \\
\hline Puerto Rico & Karso del Norte & Pleodendron macranthum & $\mathrm{CR}$ \\
\hline Puerto Rico & Karso del Sur & Zamia portoricensis & $\mathrm{CR}$ \\
\hline Puerto Rico & Maricao y Susúa & Calyptranthes portoricensis & EN \\
\hline Puerto Rico & Maricao y Susúa & Xylosma pachyphyllum & $\mathrm{CR}$ \\
\hline Puerto Rico & Mona y Monito & Eleutherodactylus monensis & VU \\
\hline Puerto Rico & Mona y Monito & Sphaerodactylus micropithecus & EN \\
\hline Puerto Rico & Mona y Monito & Typhlops monensis & EN \\
\hline Puerto Rico & Sabana Seca * & Eleutherodactylus juanariveroi & $\mathrm{CR}$ \\
\hline Puerto Rico & Salinas de Punta Cucharas & Trichilia triacantha & $\mathrm{CR}$ \\
\hline Puerto Rico & Sierra de Pandura * & Eleutherodactylus cooki & VU \\
\hline St Lucia & Government Forest Reserve * & Leucopeza semperi & $\mathrm{CR}$ \\
\hline St Lucia & Point Sables & Liophis ornatus & EN \\
\hline
\end{tabular}

* Global AZE site 2010 update

Sites regularly supporting significant populations of restricted-range species are global conservation priorities because there are few or no other sites in the world where conservation action for these species can be taken. However, there are no quantitative data for restricted-range species (other than birds) in the hotspot and thus this criterion could not be applied.

The main threats to the terrestrial biodiversity of the insular Caribbean, as prioritized during the national ecosystem profiling workshops in order of highly significant regional threat or impact are: invasive species; residential and commercial development; severe weather events and global climate change; agricultural expansion and intensification; overexploitation of natural resources; mining and energy production; pollution; transportation; and geological events. There is a complex mix of interacting socioeconomic, political, cultural and environmental factors that are driving environmental change and threatening biodiversity (and thus the KBAs) in the insular Caribbean. Principal among these are the increasing human population and material consumption, poverty and inequitable access to resources, the inherent economic and environmental vulnerability of the islands to external forces, such as changes in global trade regimes, and climate change. Some of these, such as poverty, are local or national issues, while others, such as climate change, require attention at the global level. All these drivers can be either exacerbated or mitigated by public policies and institutional arrangements, at national, regional and international levels.

A number of constraints need to be overcome to address the environmental threats and achieve more effective conservation of biodiversity and ecosystem services. The main ones discussed during the national workshops and consultations in order of highly significant regional barrier are: weak and ineffective policy; poor land-use planning; limited capacity and resources for biodiversity conservation; inefficient institutional frameworks; poor participation by stakeholders; limited technical and scientific knowledge for decision-making; and lack of awareness of biodiversity and ecosystem services (particularly their value) among decision-makers and the general public.

Most countries have significantly updated, or are in the process of updating (e.g. Haiti and St.Vincent), their policies and legislation on biodiversity, environmental management and sustainable development while their obligations under international agreements have helped drive this process (Brown et al. 2007). However, there exists significant variation among countries with regard to comprehensiveness and effectiveness, particularly concerning the protection of threatened biodiversity and ecosystems (BirdLife International 2008), and there is a need for specific analyses of "gaps" in legislation and policies, which very few countries have undertaken (an exception being Jamaica [NEPA 2003]). Overall, national public policy frameworks for environmental 
management remain largely oriented toward control, regulation and a reactive approach to environmental issues, although new approaches and instruments, including environment service markets, have begun to be promoted by some donors, governments and NGOs as means of changing destructive patterns of behavior.

Environmental policy in the Caribbean tends mostly to address environmental issues and impacts rather than their underlying root causes/drivers, such as human population increase. In the case of climate change, Caribbean countries do not consider themselves to be net contributors and therefore policy responses are largely limited to adaptation. All the countries in the hotspot are active participants in the main multilateral environmental agreements. All are signatories to the three "Rio Conventions" - the UN Convention on Biological Diversity, UN Convention to Combat Desertification, and the UN Framework Convention on Climate Change - and most are members of the other key biodiversity related agreements, such as the Ramsar Convention, the World Heritage Convention and the Convention on International Trade in Endangered Species, but not the Convention on Migratory Species.

At the regional level, the main agreement is the Convention for the Protection and Development of the Marine Environment of the Wider Caribbean Region (Cartagena Convention) and its three Protocols. These together constitute the only legal instrument for regional cooperation on environmental issues for the wider Caribbean, although not all signatory countries are properly meeting their commitments, e.g. required legislation not enacted, management plans not developed, and biodiversity action plans not in place.

Key Biodiversity Areas can achieve the following:

(i) Help Caribbean nations honour their commitments to multilateral environmental agreements

(ii) Provide the basis for protected area gap analyses (as is the case in Haiti where KBAs are being used in the development of the national system for protected areas plan)

(iii) Provide a prioritized framework within which to monitor the status of biodiversity in the region.

\section{REFERENCES}

Alliance for Zero Extinction (2010). 2010 AZE Update. www. zeroextinction.org. Accessed on January 2011.

BirdLife International (2008). Important Bird Areas in the Caribbean: key sites for conservation. Cambridge, UK: BirdLife International (BirdLife Conservation Series No. 15), 348pp.

Brown, N., T. Geoghegan \& Y. Renard (2007). A Situation Analysis for the Wider Caribbean. Gland, Switzerland: IUCN, $\mathrm{x}+52 \mathrm{pp}$.

Brown, J.H. \& M.V. Lomolino (1998). Biogeography-2 $2^{\text {nd }}$ Edition. Sinauer Associates, Inc. Publishers, Sunderland, Massachusetts, 691pp.

Dow, W., K. Eckert, M. Palmer \& P. Kramer (2007). An Atlas of SeaTurtle Nesting Habitat for the Wider Caribbean Region. The Wider Caribbean Sea Turtle Conservation Network and The Nature Conservancy. WIDECAST Technical Report No. 6. Beaufort, North Carolina, USA, 267pp.

NEPA (2003). Gap analysis of relevant policies. Report no. 2. Policy Analysis Report, prepared by Pauline McHardy. National Environment and Planning Agency, Kingston, Jamaica, 86pp (unpublished report).

Smith, M.L., S.B. Hedges, W. Buck, A. Hemphill, S. Inchaustegui, M.A. Ivie, D. Martina, M. Maunder, \& J.F. Ortega (2004). Caribbean Islands, pp.112-118. In: Mittermeier, R.A., P.R Gil, M. Hoffmann, J. Pilgrim, T. Brooks, C.G. Mittermeier, J. Lamoreux \& G.A.B. da Fonseca (2004). Hotspots Revisited: Earth's Biologically Richest and Most Endangered Ecoregions. Mexico City (Mexico): CEMEX, 392pp.

Stattersfield, A.J., M.J. Crosby, A.J. Long \& D.C. Wege (1998). Endemic Bird Areas of The World: Priorities for Biodiversity Conservation. Cambridge, U.K.: BirdLife International, 846pp.

Wege, D.C., D. Ryan, N. Varty, V. Anadón-Irizarry \& A. Pérez-Leroux (2010). Ecosystem Profile: the Caribbean Islands Biodiversity Hotspot. Washington, DC: Critical Ecosystem Partnership Fund,153pp (unpublished report). 
Appendix 1. Key Biodiversity Areas in the Caribbean Islands Hotspot

\begin{tabular}{|c|c|c|c|}
\hline Country & Key Biodiversity Area & Species scientific name & $\begin{array}{l}\text { IUCN Red List } \\
\text { Category }\end{array}$ \\
\hline \multirow[t]{8}{*}{ Anguilla (to UK) } & Cove Pond & Alsophis rijersmai & EN \\
\hline & Eastern Anguilla & Alsophis rijersmai & EN \\
\hline & & Iguana delicatissima & VU \\
\hline & Grey Pond & Alsophis rijersmai & EN \\
\hline & Katouche Canyon & Alsophis rijersmai & EN \\
\hline & & Iguana delicatissima & $\mathrm{VU}$ \\
\hline & Long Pond & Alsophis rijersmai & EN \\
\hline & Scrub Island & Alsophis rijersmai & EN \\
\hline \multirow[t]{14}{*}{ Antigua and Barbuda } & Bethesda Dam & Dendrocygna arborea & $\mathrm{VU}$ \\
\hline & Christian Cove & Dendrocygna arborea & VU \\
\hline & Codrington Lagoon and the Creek & Dendrocygna arborea & VU \\
\hline & Fitches Creek Bay & Dendrocygna arborea & VU \\
\hline & Hanson's Bay - Flashes & Dendrocygna arborea & VU \\
\hline & McKinnons Salt Pond & Dendrocygna arborea & VU \\
\hline & Offshore Islands & Alsophis antiguae & $\mathrm{CR}$ \\
\hline & & Dendrocygna arborea & $\mathrm{VU}$ \\
\hline & & Eretmochelys imbricata & $\mathrm{CR}$ \\
\hline & & Guaiacum officinale & EN \\
\hline & Potworks Dam & Dendrocygna arborea & $\mathrm{VU}$ \\
\hline & Valley Church Bay & Dendrocygna arborea & $\mathrm{VU}$ \\
\hline & Wallings Forest & Guaiacum officinale & EN \\
\hline & & Swietenia mahagoni & EN \\
\hline \multirow[t]{2}{*}{ Aruba (to Netherlands) } & Arikok National Park & Crotalus unicolor & $\mathrm{CR}$ \\
\hline & & Leptonycteris curasoae & VU \\
\hline \multirow[t]{30}{*}{ Bahamas } & Allan's Cays & Cyclura cychlura & VU \\
\hline & Bitter Guana Cay & Cyclura cychlura & VU \\
\hline & Booby Cay & Cyclura carinata & CR \\
\hline & Cat Island Wetlands & Dendrocygna arborea & VU \\
\hline & Cay Sal & Caretta caretta & EN \\
\hline & East Plana Key & Geocapromys ingrahami & $\mathrm{VU}$ \\
\hline & Exuma Cays Land and Sea Park & Cyclura cychlura & VU \\
\hline & & Geocapromys ingrahami & VU \\
\hline & Graham's Harbour & Cyclura rileyi & EN \\
\hline & Great Inagua & Dendrocygna arborea & $\mathrm{VU}$ \\
\hline & Green Cay & Cyclura rileyi & EN \\
\hline & Grog Pond & Dendrocygna arborea & VU \\
\hline & Guana Cays & Cyclura rileyi & EN \\
\hline & Harrold and Wilson Ponds National Park & Tachycineta cyaneoviridis & EN \\
\hline & Lee Stocking Island & Dendrocygna arborea & VU \\
\hline & Long Island and Hog Cay & Dendrocygna arborea & VU \\
\hline & Low Cay, High Cay and Sandy Hook & Cyclura rileyi & EN \\
\hline & Lucayan National Park & Tachycineta cyaneoviridis & EN \\
\hline & Mangrove Cay & Cyclura cychlura & VU \\
\hline & Red Bays & Tachycineta cyaneoviridis & EN \\
\hline & San Andros Pond & Dendrocygna arborea & $\mathrm{VU}$ \\
\hline & & Tachycineta cyaneoviridis & EN \\
\hline & San Salvador & Eugenia crenata & VU \\
\hline & South Andros Island & Cyclura cychlura & VU \\
\hline & & Trachemys terrapen & VU \\
\hline & Southern Great Lake & Cyclura rileyi & EN \\
\hline & Stafford Creek to Andros Town & Cyclura cychlura & VU \\
\hline & & Tachycineta cyaneoviridis & EN \\
\hline & White Bay, Noddy, North Adderley and Leaf Cays & Cyclura cychlura & VU \\
\hline & White Cay & Cyclura rileyi & EN \\
\hline \multirow[t]{4}{*}{ Barbados } & Bath Beach & Eretmochelys imbricata & $\mathrm{CR}$ \\
\hline & Hilton Beach & Eretmochelys imbricata & CR \\
\hline & Scotland District & Dermochelys coriacea & $\mathrm{CR}$ \\
\hline & & Liophis perfuscus & EN \\
\hline
\end{tabular}




\begin{tabular}{|c|c|c|c|}
\hline Country & Key Biodiversity Area & Species scientific name & $\begin{array}{l}\text { IUCN Red List } \\
\text { Category }\end{array}$ \\
\hline & & Myotis martiniquensis & VU \\
\hline & South Coast Beaches & Eretmochelys imbricata & $\mathrm{CR}$ \\
\hline & West Coast Beaches & Eretmochelys imbricata & $\mathrm{CR}$ \\
\hline \multirow[t]{5}{*}{ Bonaire } & Dos Pos, Bonaire & Amazona barbadensis & VU \\
\hline & Lac Bay, Bonaire & Amazona barbadensis & VU \\
\hline & Washikemba-Fontein-Onima, Bonaire & Amazona barbadensis & VU \\
\hline & Washington-Slagbaai National Park, Bonaire & Amazona barbadensis & VU \\
\hline & & Leptonycteris curasoae & VU \\
\hline \multirow[t]{13}{*}{ British Virgin Islands } & Anegada Island & Acacia anegadensis & $\mathrm{CR}$ \\
\hline & & Cordia rupicola & $\mathrm{CR}$ \\
\hline & & Cyclura pinguis & $\mathrm{CR}$ \\
\hline & & Leptocereus quadricostatus & $\mathrm{CR}$ \\
\hline & & Metastelma anegadense & $\mathrm{CR}$ \\
\hline & Guana Island & Cyclura pinguis & $\mathrm{CR}$ \\
\hline & Little Thatch Island & Cyclura pinguis & $\mathrm{CR}$ \\
\hline & Necker Island & Cyclura pinguis & $\mathrm{CR}$ \\
\hline & Sage Mountain Area & Eleutherodactylus schwartzi & EN \\
\hline & Virgin Gorda & Calyptranthes kiaerskovii & $\mathrm{CR}$ \\
\hline & & Eleutherodactylus schwartzi & EN \\
\hline & & Machaonia woodburyana & $\mathrm{CR}$ \\
\hline & & Maytenus cymosa & EN \\
\hline \multirow[t]{12}{*}{ Cayman Islands (to UK) } & Bluff Forest & Cedrela odorata & VU \\
\hline & & Cyclura nubila & VU \\
\hline & Booby Pond Nature Reserve & Cyclura nubila & VU \\
\hline & & Dendrocygna arborea & VU \\
\hline & Botanic Park and Salina Reserve & Cyclura lewisi & $\mathrm{CR}$ \\
\hline & & Dendrocygna arborea & VU \\
\hline & Central Mangrove Wetland & Dendrocygna arborea & VU \\
\hline & Crown Wetlands & Cyclura nubila & VU \\
\hline & & Dendrocygna arborea & VU \\
\hline & Eastern Dry Forest & Cyclura lewisi & $\mathrm{CR}$ \\
\hline & Franklin's Forest & Cyclura lewisi & $\mathrm{CR}$ \\
\hline & Sparrowhawk Hill & Cyclura nubila & VU \\
\hline \multirow[t]{23}{*}{ Cuba } & Alejandro de Humboldt & Accipiter gundlachi & EN \\
\hline & & Aratinga euops & VU \\
\hline & & Campephilus principalis & $\mathrm{CR}$ \\
\hline & & Chondrohierax wilsonii & $\mathrm{CR}$ \\
\hline & & Geotrygon caniceps & VU \\
\hline & & Starnoenas cyanocephala & EN \\
\hline & & Tyrannus cubensis & EN \\
\hline & Alturas de Banao & Accipiter gundlachi & EN \\
\hline & & Aratinga euops & VU \\
\hline & & Geotrygon caniceps & VU \\
\hline & Cayería Centro-Oriental de Villa Clara & Dendrocygna arborea & VU \\
\hline & $\begin{array}{l}\text { Cayo Sabinal, Ballenatos y Manglares de la Bahía } \\
\text { de Nuevitas }\end{array}$ & Colaptes fernandinae & VU \\
\hline & & Dendrocygna arborea & VU \\
\hline & Cayos Romano-Cruz-Megano Grande & Accipiter gundlachi & EN \\
\hline & & Dendrocygna arborea & VU \\
\hline & Ciénaga de Lanier y Sur de la Isla de la Juventud & Dendrocygna arborea & VU \\
\hline & & Tyrannus cubensis & EN \\
\hline & Ciénaga de Zapata & Accipiter gundlachi & EN \\
\hline & & Aratinga euops & VU \\
\hline & & Colaptes fernandinae & VU \\
\hline & & Cyanolimnas cerverai & $\mathrm{CR}$ \\
\hline & & Dendrocygna arborea & VU \\
\hline & & Ferminia cerverai & EN \\
\hline
\end{tabular}




\begin{tabular}{|c|c|c|c|}
\hline Country & Key Biodiversity Area & Species scientific name & $\begin{array}{l}\text { IUCN Red List } \\
\text { Category }\end{array}$ \\
\hline & & Geotrygon caniceps & VU \\
\hline & & Starnoenas cyanocephala & EN \\
\hline & & Torreornis inexpectata & EN \\
\hline & & Tyrannus cubensis & EN \\
\hline & Delta del Cauto & Accipiter gundlachi & EN \\
\hline & & Aratinga euops & VU \\
\hline & & Colaptes fernandinae & VU \\
\hline & & Dendrocygna arborea & VU \\
\hline & Delta del Mayarí & Accipiter gundlachi & EN \\
\hline & & Dendrocygna arborea & VU \\
\hline & Desembarco del Granma & Accipiter gundlachi & EN \\
\hline & & Colaptes fernandinae & VU \\
\hline & & Dendrocygna arborea & VU \\
\hline & & Geotrygon caniceps & VU \\
\hline & & Starnoenas cyanocephala & EN \\
\hline & & Tyrannus cubensis & EN \\
\hline & Gibara & Accipiter gundlachi & EN \\
\hline & & Dendrocygna arborea & VU \\
\hline & & Geotrygon caniceps & VU \\
\hline & Gran Humedal del Norte de Ciego de Ávila & Accipiter gundlachi & EN \\
\hline & & Aratinga euops & VU \\
\hline & & Dendrocygna arborea & VU \\
\hline & & Starnoenas cyanocephala & EN \\
\hline & & Torreornis inexpectata & EN \\
\hline & Gran Piedra - Pico Mogote & Accipiter gundlachi & EN \\
\hline & & Geotrygon caniceps & VU \\
\hline & & Tyrannus cubensis & EN \\
\hline & Guanahacabibes & Accipiter gundlachi & EN \\
\hline & & Dendrocygna arborea & VU \\
\hline & & Starnoenas cyanocephala & EN \\
\hline & & Tyrannus cubensis & EN \\
\hline & Hatibonico - Baitiquirí - Imías & Accipiter gundlachi & EN \\
\hline & & Colaptes fernandinae & VU \\
\hline & & Geotrygon caniceps & VU \\
\hline & & Torreornis inexpectata & EN \\
\hline & Humedal Sur de Pinar del Río & Dendrocygna arborea & VU \\
\hline & Humedal Sur de Sancti Spiritus & Dendrocygna arborea & VU \\
\hline & La Mensura & Accipiter gundlachi & EN \\
\hline & & Aratinga euops & VU \\
\hline & & Geotrygon caniceps & VU \\
\hline & Las Picúa- Cayo del Cristo & Dendrocygna arborea & VU \\
\hline & Limones-Tuabaquey & Accipiter gundlachi & EN \\
\hline & & Aratinga euops & VU \\
\hline & & Colaptes fernandinae & VU \\
\hline & & Geotrygon caniceps & VU \\
\hline & & Starnoenas cyanocephala & EN \\
\hline & Mil Cumbres & Accipiter gundlachi & EN \\
\hline & & Aratinga euops & VU \\
\hline & & Colaptes fernandinae & $\mathrm{VU}$ \\
\hline & & Geotrygon caniceps & VU \\
\hline & & Starnoenas cyanocephala & EN \\
\hline & & Tyrannus cubensis & EN \\
\hline & Pico Cristal & Accipiter gundlachi & EN \\
\hline & & Aratinga euops & VU \\
\hline & & Geotrygon caniceps & VU \\
\hline & & Tyrannus cubensis & EN \\
\hline & Río Máximo & Accipiter gundlachi & EN \\
\hline & & Colaptes fernandinae & VU \\
\hline & & Dendrocygna arborea & VU \\
\hline & & Geotrygon caniceps & VU \\
\hline
\end{tabular}




\begin{tabular}{|c|c|c|c|}
\hline Country & Key Biodiversity Area & Species scientific name & $\begin{array}{c}\text { IUCN Red List } \\
\text { Category }\end{array}$ \\
\hline & Siboney - Juticí & Accipiter gundlachi & EN \\
\hline & Sierra del Chorrillo & Accipiter gundlachi & EN \\
\hline & & Aratinga euops & VU \\
\hline & & Colaptes fernandinae & VU \\
\hline & & Dendrocygna arborea & VU \\
\hline & & Starnoenas cyanocephala & EN \\
\hline & & Tyrannus cubensis & EN \\
\hline & Sierra del Rosario & Accipiter gundlachi & EN \\
\hline & & Colaptes fernandinae & VU \\
\hline & & Geotrygon caniceps & VU \\
\hline & & Starnoenas cyanocephala & EN \\
\hline & & Tyrannus cubensis & EN \\
\hline & Topes de Collantes & Accipiter gundlachi & EN \\
\hline & & Aratinga euops & VU \\
\hline & & Geotrygon caniceps & VU \\
\hline & Turquino-Bayamesa & Accipiter gundlachi & EN \\
\hline & & Catharus bicknelli & VU \\
\hline & & Colaptes fernandinae & VU \\
\hline & & Geotrygon caniceps & VU \\
\hline & & Pterodroma hasitata & EN \\
\hline & & Starnoenas cyanocephala & EN \\
\hline & & Tyrannus cubensis & EN \\
\hline \multirow[t]{20}{*}{ Dominica } & Cochrane & Dactylolejeunea acanthifolia & VU \\
\hline & & Inga dominicensis & VU \\
\hline & & Tabernaemontana oppositifolia & VU \\
\hline & Morne Diablotin National Park & Amazona arausiaca & VU \\
\hline & & Amazona imperialis & EN \\
\hline & & Eleutherodactylus amplinympha & EN \\
\hline & & Iguana delicatissima & VU \\
\hline & & Turdus Iherminieri & VU \\
\hline & Morne Trois Pitons National Park & Amazona arausiaca & VU \\
\hline & & Amazona imperialis & EN \\
\hline & & Dactylolejeunea acanthifolia & VU \\
\hline & & Eleutherodactylus amplinympha & EN \\
\hline & & Inga dominicensis & VU \\
\hline & & Leptodactylus fallax & $\mathrm{CR}$ \\
\hline & & Phycolepidozia exigua & $\mathrm{CR}$ \\
\hline & & Pouteria pallida & EN \\
\hline & & Pouteria semecarpifolia & VU \\
\hline & & Tabernaemontana oppositifolia & VU \\
\hline & & Turdus Iherminieri & VU \\
\hline & Point Des Foux & Iguana delicatissima & VU \\
\hline \multirow[t]{14}{*}{ Dominican Republic } & Arroyo Lebrón, El Seybo & Stahlia monosperma & EN \\
\hline & Bahía de las Calderas & Cyclura cornuta & VU \\
\hline & & Dendrocygna arborea & VU \\
\hline & & Senna domingensis & VU \\
\hline & Bahoruco Oriental & Amazona ventralis & VU \\
\hline & & Antirhea radiata & VU \\
\hline & & Aratinga chloroptera & VU \\
\hline & & Calyptophilus frugivorus & VU \\
\hline & & Corvus leucognaphalus & VU \\
\hline & & Eleutherodactylus armstrongi & EN \\
\hline & & Eleutherodactylus audanti & VU \\
\hline & & Eleutherodactylus heminota & EN \\
\hline & & Eleutherodactylus hypostenor & EN \\
\hline & & Eleutherodactylus leoncei & $\mathrm{CR}$ \\
\hline
\end{tabular}




\begin{tabular}{|c|c|c|c|}
\hline Country & Key Biodiversity Area & Species scientific name & $\begin{array}{l}\text { IUCN Red List } \\
\text { Category }\end{array}$ \\
\hline & & Eleutherodactylus rufifemoralis & $\mathrm{CR}$ \\
\hline & & Geotrygon caniceps & VU \\
\hline & & Huertea cubensis & VU \\
\hline & & Solenodon paradoxus & EN \\
\hline & & Tachycineta euchrysea & VU \\
\hline & Cabo Engaño & Swietenia mahagoni & EN \\
\hline & Cerro Chacuey & Swietenia mahagoni & EN \\
\hline & Diego de Ocampo & Hypsiboas heilprini & VU \\
\hline & Honduras & Calyptophilus frugivorus & VU \\
\hline & & Guaiacum officinale & EN \\
\hline & & Guaiacum sanctum & EN \\
\hline & & Hypsiboas heilprini & VU \\
\hline & & Peltophryne guentheri & VU \\
\hline & & Swietenia mahagoni & EN \\
\hline & Hoyo Claro-Hoyo Azul & Amazona ventralis & VU \\
\hline & & Eleutherodactylus probolaeus & EN \\
\hline & & Eleutherodactylus ruthae & EN \\
\hline & & Guaiacum officinale & EN \\
\hline & & Guaiacum sanctum & EN \\
\hline & & Swietenia mahagoni & EN \\
\hline & Lago Enriquillo & Amazona ventralis & VU \\
\hline & & Corvus leucognaphalus & VU \\
\hline & & Crocodylus acutus & VU \\
\hline & & Cyclura cornuta & VU \\
\hline & & Cyclura ricordi & $\mathrm{CR}$ \\
\hline & & Dendrocygna arborea & VU \\
\hline & & Eleutherodactylus pictissimus & VU \\
\hline & & Guaiacum officinale & EN \\
\hline & & Guaiacum sanctum & EN \\
\hline & & Peltophryne guentheri & VU \\
\hline & & Senna domingensis & VU \\
\hline & & Trachemys decorata & VU \\
\hline & Laguna Bávaro & Eleutherodactylus pictissimus & VU \\
\hline & & Eleutherodactylus probolaeus & EN \\
\hline & & Eleutherodactylus ruthae & EN \\
\hline & Laguna Cabral & Aratinga chloroptera & VU \\
\hline & & Cyclura cornuta & $\mathrm{VU}$ \\
\hline & & Dendrocygna arborea & VU \\
\hline & & Eleutherodactylus pictissimus & VU \\
\hline & & Peltophryne guentheri & VU \\
\hline & & Senna domingensis & VU \\
\hline & & Trachemys decorata & VU \\
\hline & Laguna Limón & Dendrocygna arborea & VU \\
\hline & Loma Guaconejo & Catharus bicknelli & VU \\
\hline & & Osteopilus pulchrilineatus & EN \\
\hline & & Osteopilus vastus & EN \\
\hline & & Plagiodontia aedium & EN \\
\hline & & Solenodon paradoxus & EN \\
\hline & Loma Isabel de Torres & Osteopilus pulchrilineatus & EN \\
\hline & & Tabernaemontana oppositifolia & VU \\
\hline & Loma La Humeadora & Amazona ventralis & VU \\
\hline & & Antirhea sintenisii & VU \\
\hline & & Aratinga chloroptera & VU \\
\hline & & Ateleia gummifera & EN \\
\hline & & Catharus bicknelli & VU \\
\hline & & Celestus anelpistus & $\mathrm{CR}$ \\
\hline
\end{tabular}




\begin{tabular}{|c|c|c|c|}
\hline Country & Key Biodiversity Area & Species scientific name & $\begin{array}{l}\text { IUCN Red List } \\
\text { Category }\end{array}$ \\
\hline & & Eleutherodactylus auriculatoides & EN \\
\hline & & Eleutherodactylus minutus & EN \\
\hline & & Eleutherodactylus patriciae & EN \\
\hline & & Eleutherodactylus pituinus & EN \\
\hline & & Eleutherodactylus pituinus & EN \\
\hline & & Eleutherodactylus schmidti & $\mathrm{CR}$ \\
\hline & & Hypsiboas heilprini & VU \\
\hline & & Osteopilus vastus & EN \\
\hline & & Pimenta cainitoides & VU \\
\hline & & Turdus swalesi & EN \\
\hline & Loma Nalga de Maco y Río Limpio & Amazona ventralis & VU \\
\hline & & Aratinga chloroptera & VU \\
\hline & & Calyptophilus frugivorus & VU \\
\hline & & Catharus bicknelli & VU \\
\hline & & Coccyzus rufigularis & EN \\
\hline & & Corvus leucognaphalus & $\mathrm{VU}$ \\
\hline & & Eleutherodactylus audanti & VU \\
\hline & & Eleutherodactylus auriculatoides & EN \\
\hline & & Eleutherodactylus montanus & EN \\
\hline & & Eleutherodactylus schmidti & $\mathrm{CR}$ \\
\hline & & Eleutherodactylus wetmorei & VU \\
\hline & & Hypsiboas heilprini & VU \\
\hline & & Lasiurus minor & VU \\
\hline & & Osteopilus pulchrilineatus & EN \\
\hline & & Podocarpus hispaniolensis & EN \\
\hline & & Tachycineta euchrysea & VU \\
\hline & & Xenoligea montana & $\mathrm{VU}$ \\
\hline & Loma Quita Espuela & Amazona ventralis & VU \\
\hline & & Aratinga chloroptera & VU \\
\hline & & Catharus bicknelli & VU \\
\hline & & Eleutherodactylus schmidti & $\mathrm{CR}$ \\
\hline & & Hypsiboas heilprini & VU \\
\hline & & Osteopilus pulchrilineatus & EN \\
\hline & & Osteopilus vastus & EN \\
\hline & & Plagiodontia aedium & EN \\
\hline & & Solenodon paradoxus & EN \\
\hline & Los Haitises & Abarema abbottii & VU \\
\hline & & Amazona ventralis & VU \\
\hline & & Buteo ridgwayi & $\mathrm{CR}$ \\
\hline & & Catharus bicknelli & VU \\
\hline & & Corvus leucognaphalus & VU \\
\hline & & Huertea cubensis & VU \\
\hline & & Hypsiboas heilprini & VU \\
\hline & & Osteopilus pulchrilineatus & EN \\
\hline & & Osteopilus vastus & EN \\
\hline & & Plagiodontia aedium & EN \\
\hline & & Sideroxylon dominicanum & VU \\
\hline & & Solenodon paradoxus & EN \\
\hline & Manglares de Estero Balsa & Crocodylus acutus & $\mathrm{VU}$ \\
\hline & & Cyclura cornuta & VU \\
\hline & & Guaiacum officinale & EN \\
\hline & & Guaiacum sanctum & EN \\
\hline & & Peltophryne guentheri & VU \\
\hline & Manglares del Bajo Yuna & Plagiodontia aedium & EN \\
\hline & Parque Nacional Armando Bermúdez & Amazona ventralis & VU \\
\hline & & Aratinga chloroptera & $\mathrm{VU}$ \\
\hline & & Calyptophilus frugivorus & VU \\
\hline
\end{tabular}




\begin{tabular}{|c|c|c|c|}
\hline Country & Key Biodiversity Area & Species scientific name & $\begin{array}{l}\text { IUCN Red List } \\
\text { Category }\end{array}$ \\
\hline & & Catharus bicknelli & VU \\
\hline & & Coccyzus rufigularis & EN \\
\hline & & Corvus leucognaphalus & VU \\
\hline & & Eleutherodactylus audanti & VU \\
\hline & & Eleutherodactylus auriculatoides & EN \\
\hline & & Eleutherodactylus haitianus & EN \\
\hline & & Eleutherodactylus minutus & EN \\
\hline & & Eleutherodactylus montanus & EN \\
\hline & & Eleutherodactylus patriciae & EN \\
\hline & & Eleutherodactylus pituinus & EN \\
\hline & & Eleutherodactylus ruthae & EN \\
\hline & & Eleutherodactylus schmidti & CR \\
\hline & & Hypsiboas heilprini & VU \\
\hline & & Lasiurus minor & VU \\
\hline & & Loxia megaplaga & EN \\
\hline & & Osteopilus vastus & EN \\
\hline & & Plagiodontia aedium & EN \\
\hline & & Podocarpus aristulatus & VU \\
\hline & & Podocarpus hispaniolensis & EN \\
\hline & & Solenodon paradoxus & EN \\
\hline & & Tachycineta euchrysea & VU \\
\hline & & Turdus swalesi & EN \\
\hline & & Xenoligea montana & VU \\
\hline & Parque Nacional del Este & Amazona ventralis & VU \\
\hline & & Catharus bicknelli & VU \\
\hline & & Corvus leucognaphalus & VU \\
\hline & & Cyclura cornuta & VU \\
\hline & & Eleutherodactylus probolaeus & EN \\
\hline & & Guaiacum officinale & EN \\
\hline & & Guaiacum sanctum & EN \\
\hline & & Plagiodontia aedium & EN \\
\hline & & Solenodon paradoxus & EN \\
\hline & & Zanthoxylum flavum & VU \\
\hline & Parque Nacional Jaragua & Amazona ventralis & VU \\
\hline & & Aratinga chloroptera & VU \\
\hline & & Corvus leucognaphalus & VU \\
\hline & & Cyclura cornuta & VU \\
\hline & & Cyclura ricordi & $\mathrm{CR}$ \\
\hline & & Dendrocygna arborea & VU \\
\hline & & Ekmanianthe longiflora & EN \\
\hline & & Eleutherodactylus alcoae & EN \\
\hline & & Guaiacum sanctum & EN \\
\hline & & Pimenta haitiensis & VU \\
\hline & & Plagiodontia aedium & EN \\
\hline & & Pseudophoenix ekmanii & $\mathrm{CR}$ \\
\hline & & Solenodon paradoxus & EN \\
\hline & & Swietenia mahagoni & EN \\
\hline & & Trachemys decorata & VU \\
\hline & Parque Nacional Jose del Carmen Ramirez & Eleutherodactylus audanti & VU \\
\hline & & Eleutherodactylus auriculatoides & EN \\
\hline & & Eleutherodactylus montanus & EN \\
\hline & & Eleutherodactylus patriciae & EN \\
\hline & & Hypsiboas heilprini & VU \\
\hline & & Osteopilus pulchrilineatus & EN \\
\hline & & Osteopilus vastus & EN \\
\hline & Promontorio de Cabrera & Solenodon paradoxus & EN \\
\hline & Reserva Científica Ébano Verde & Antirhea sintenisii & VU \\
\hline
\end{tabular}




\begin{tabular}{|c|c|c|c|}
\hline Country & Key Biodiversity Area & Species scientific name & $\begin{array}{l}\text { IUCN Red List } \\
\text { Category }\end{array}$ \\
\hline & & Calyptophilus frugivorus & VU \\
\hline & & Cleyera bolleana & VU \\
\hline & & Eleutherodactylus auriculatoides & EN \\
\hline & & Eleutherodactylus minutus & EN \\
\hline & & Eleutherodactylus pituinus & EN \\
\hline & & Eleutherodactylus schmidti & $\mathrm{CR}$ \\
\hline & & Geotrygon caniceps & VU \\
\hline & & Hypsiboas heilprini & $\mathrm{VU}$ \\
\hline & & Juglans jamaicensis & VU \\
\hline & & Lasiurus minor & VU \\
\hline & & Osteopilus vastus & EN \\
\hline & & Podocarpus aristulatus & VU \\
\hline & & Tachycineta euchrysea & VU \\
\hline & & Xenoligea montana & VU \\
\hline & Rio Anamuya & Stahlia monosperma & EN \\
\hline & Salto de la Damajagua & Osteopilus pulchrilineatus & EN \\
\hline & & Osteopilus vastus & EN \\
\hline & & Solenodon paradoxus & EN \\
\hline & Sierra de Bahoruco & Amazona ventralis & VU \\
\hline & & Aratinga chloroptera & $\mathrm{VU}$ \\
\hline & & Calyptophilus frugivorus & VU \\
\hline & & Catharus bicknelli & VU \\
\hline & & Coccyzus rufigularis & EN \\
\hline & & Corvus leucognaphalus & VU \\
\hline & & Cyclura cornuta & VU \\
\hline & & Ekmanianthe longiflora & EN \\
\hline & & Eleutherodactylus alcoae & EN \\
\hline & & Eleutherodactylus armstrongi & EN \\
\hline & & Eleutherodactylus audanti & VU \\
\hline & & Eleutherodactylus fowleri & CR \\
\hline & & Eleutherodactylus furcyensis & $\mathrm{CR}$ \\
\hline & & Eleutherodactylus heminota & EN \\
\hline & & Eleutherodactylus hypostenor & EN \\
\hline & & Eleutherodactylus jugans & $\mathrm{CR}$ \\
\hline & & Eleutherodactylus leoncei & $\mathrm{CR}$ \\
\hline & & Eleutherodactylus nortoni & $\mathrm{CR}$ \\
\hline & & Eleutherodactylus pictissimus & VU \\
\hline & & Eleutherodactylus rufifemoralis & $\mathrm{CR}$ \\
\hline & & Eleutherodactylus wetmorei & VU \\
\hline & & Juniperus gracilior & EN \\
\hline & & Lasiurus minor & VU \\
\hline & & Loxia megaplaga & EN \\
\hline & & Osteopilus vastus & EN \\
\hline & & Plagiodontia aedium & EN \\
\hline & & Podocarpus aristulatus & $\mathrm{VU}$ \\
\hline & & Pterodroma hasitata & EN \\
\hline & & Solenodon paradoxus & EN \\
\hline & & Tachycineta euchrysea & VU \\
\hline & & Trachemys decorata & $\mathrm{VU}$ \\
\hline & & Turdus swalesi & EN \\
\hline & & Xenoligea montana & VU \\
\hline & Sierra de Neyba & Amazona ventralis & VU \\
\hline & & Calyptophilus frugivorus & VU \\
\hline & & Coccyzus rufigularis & EN \\
\hline & & Corvus leucognaphalus & VU \\
\hline & & Ekmanianthe longiflora & EN \\
\hline & & Eleutherodactylus audanti & VU \\
\hline
\end{tabular}




\begin{tabular}{|c|c|c|c|}
\hline Country & Key Biodiversity Area & Species scientific name & $\begin{array}{l}\text { IUCN Red List } \\
\text { Category }\end{array}$ \\
\hline & & Eleutherodactylus parabates & $\mathrm{CR}$ \\
\hline & & Eleutherodactylus wetmorei & VU \\
\hline & & Hypsiboas heilprini & VU \\
\hline & & Juniperus gracilior & EN \\
\hline & & Lasiurus minor & $\mathrm{VU}$ \\
\hline & & Plagiodontia aedium & EN \\
\hline & & Podocarpus aristulatus & VU \\
\hline & & Podocarpus hispaniolensis & EN \\
\hline & & Solenodon paradoxus & EN \\
\hline & & Tachycineta euchrysea & VU \\
\hline & & Turdus swalesi & EN \\
\hline & & Xenoligea montana & VU \\
\hline & Sierra Martín García & Amazona ventralis & VU \\
\hline & & Calyptophilus frugivorus & VU \\
\hline & & Catharus bicknelli & VU \\
\hline & & Cyclura cornuta & $\mathrm{VU}$ \\
\hline & & Eleutherodactylus pictissimus & VU \\
\hline & & Guaiacum officinale & EN \\
\hline & & Juniperus gracilior & EN \\
\hline & & Manilkara valenzuelana & VU \\
\hline & & Peltophryne guentheri & VU \\
\hline & & Swietenia mahagoni & EN \\
\hline & & Xenoligea montana & VU \\
\hline & & Zanthoxylum flavum & VU \\
\hline & Valle Nuevo & Amazona ventralis & VU \\
\hline & & Antirhea sintenisii & VU \\
\hline & & Aratinga chloroptera & VU \\
\hline & & Calyptophilus frugivorus & VU \\
\hline & & Catharus bicknelli & VU \\
\hline & & Cleyera bolleana & VU \\
\hline & & Cleyera vaccinioides & VU \\
\hline & & Eleutherodactylus audanti & $\mathrm{VU}$ \\
\hline & & Eleutherodactylus auriculatoides & EN \\
\hline & & Eleutherodactylus haitianus & EN \\
\hline & & Eleutherodactylus minutus & EN \\
\hline & & Eleutherodactylus montanus & EN \\
\hline & & Eleutherodactylus patriciae & EN \\
\hline & & Eleutherodactylus pituinus & EN \\
\hline & & Eleutherodactylus schmidti & $\mathrm{CR}$ \\
\hline & & Hypsiboas heilprini & VU \\
\hline & & Juniperus gracilior & EN \\
\hline & & Lasiurus minor & VU \\
\hline & & Loxia megaplaga & EN \\
\hline & & Osteopilus vastus & EN \\
\hline & & Plagiodontia aedium & EN \\
\hline & & Podocarpus aristulatus & VU \\
\hline & & Podocarpus hispaniolensis & EN \\
\hline & & Solenodon paradoxus & EN \\
\hline & & Tachycineta euchrysea & VU \\
\hline & & Turdus swalesi & EN \\
\hline & & Xenoligea montana & VU \\
\hline \multirow[t]{6}{*}{ Grenada } & Bathway Beach & Dermochelys coriacea & CR \\
\hline & Beausejour/Grenville Vale & Leptotila wellsi & $\mathrm{CR}$ \\
\hline & Grand Etang & Pristimantis euphronides & EN \\
\hline & Levera Beach & Dermochelys coriacea & $\mathrm{CR}$ \\
\hline & Mount Hartman & Leptotila wellsi & $\mathrm{CR}$ \\
\hline & Mount Saint Catherine & Pristimantis euphronides & EN \\
\hline
\end{tabular}




\begin{tabular}{|c|c|c|c|}
\hline Country & Key Biodiversity Area & Species scientific name & $\begin{array}{l}\text { IUCN Red List } \\
\text { Category }\end{array}$ \\
\hline & Perseverance & Leptotila wellsi & $\mathrm{CR}$ \\
\hline & Woodford & Leptotila wellsi & $\mathrm{CR}$ \\
\hline & Woodlands & Leptotila wellsi & $\mathrm{CR}$ \\
\hline \multirow[t]{16}{*}{ Guadeloupe } & $\begin{array}{l}\text { Falaises Nord et Îlet de Vieux-Fort de Marie- } \\
\text { Galante }\end{array}$ & Guaiacum officinale & EN \\
\hline & Gallery Forest of Baie-Mahault & Chiroderma improvisum & VU \\
\hline & & Eptesicus guadeloupensis & VU \\
\hline & Grand Cul-de-Sac Marin's eastern coastline & Turdus Iherminieri & VU \\
\hline & îlets de la Petite-Terre & Guaiacum officinale & EN \\
\hline & & Iguana delicatissima & VU \\
\hline & La Désirade & Iguana delicatissima & VU \\
\hline & Massif forestier de l'île de Basse-Terre & Eleutherodactylus barlagnei & EN \\
\hline & & Eleutherodactylus pinchoni & EN \\
\hline & & Eptesicus guadeloupensis & VU \\
\hline & & Iguana delicatissima & VU \\
\hline & & Myotis dominicensis & VU \\
\hline & & Sturnira thomasi & VU \\
\hline & & Turdus Iherminieri & VU \\
\hline & Pointe des Châteaux & Guaiacum officinale & EN \\
\hline & Trois-Ilets \& Folle Anse de Marie-Galante & Eretmochelys imbricata & $\mathrm{CR}$ \\
\hline \multirow[t]{37}{*}{ Haiti } & Cavaillon & Attalea crassispatha & $\mathrm{CR}$ \\
\hline & Dame-Marie & Eleutherodactylus caribe & $\mathrm{CR}$ \\
\hline & & Eleutherodactylus paulsoni & $\mathrm{CR}$ \\
\hline & & Eleutherodactylus pictissimus & VU \\
\hline & & Hypsiboas heilprini & VU \\
\hline & Dubedou & Ekmanianthe longiflora & EN \\
\hline & Fond des Nêgres & Attalea crassispatha & $\mathrm{CR}$ \\
\hline & Ile de la Tortue Forest & Eleutherodactylus warreni & CR \\
\hline & Ilet Bas limbe & Cyclura cornuta & $\mathrm{VU}$ \\
\hline & Lac Azuéi & Crocodylus acutus & VU \\
\hline & & Peltophryne guentheri & VU \\
\hline & & Trachemys decorata & VU \\
\hline & Lagon-aux-Boeufs & Crocodylus acutus & VU \\
\hline & & Dendrocygna arborea & VU \\
\hline & & Peltophryne guentheri & VU \\
\hline & Citadelle-Sans Souci-Ramier & Amazona ventralis & VU \\
\hline & & Aratinga chloroptera & VU \\
\hline & & Eleutherodactylus poolei & $\mathrm{CR}$ \\
\hline & & Eleutherodactylus schmidti & $\mathrm{CR}$ \\
\hline & & Eleutherodactylus wetmorei & VU \\
\hline & & Hypsiboas heilprini & VU \\
\hline & & Osteopilus pulchrilineatus & EN \\
\hline & & Peltophryne guentheri & VU \\
\hline & Plaisance & Celestus warreni & $\mathrm{CR}$ \\
\hline & & Cyclura cornuta & VU \\
\hline & & Eleutherodactylus wetmorei & VU \\
\hline & & Hypsiboas heilprini & VU \\
\hline & & Osteopilus pulchrilineatus & EN \\
\hline & & Osteopilus vastus & EN \\
\hline & & Peltophryne guentheri & VU \\
\hline & Massif de la Hotte & Calyptophilus frugivorus & VU \\
\hline & & Calyptranthes ekmanii & VU \\
\hline & & Catalpa brevipes & VU \\
\hline & & Catharus bicknelli & VU \\
\hline & & Eleutherodactylus amadeus & $\mathrm{CR}$ \\
\hline & & Eleutherodactylus apostates & $\mathrm{CR}$ \\
\hline & & Eleutherodactylus audanti & VU \\
\hline
\end{tabular}




\begin{tabular}{|c|c|c|c|}
\hline Country & Key Biodiversity Area & Species scientific name & $\begin{array}{l}\text { IUCN Red List } \\
\text { Category }\end{array}$ \\
\hline & & Eleutherodactylus bakeri & $\mathrm{CR}$ \\
\hline & & Eleutherodactylus brevirostris & $\mathrm{CR}$ \\
\hline & & Eleutherodactylus chlorophenax & $\mathrm{CR}$ \\
\hline & & Eleutherodactylus corona & $\mathrm{CR}$ \\
\hline & & Eleutherodactylus counouspeus & EN \\
\hline & & Eleutherodactylus dolomedes & $\mathrm{CR}$ \\
\hline & & Eleutherodactylus eunaster & $\mathrm{CR}$ \\
\hline & & Eleutherodactylus glandulifer & CR \\
\hline & & Eleutherodactylus glaphycompus & EN \\
\hline & & Eleutherodactylus heminota & EN \\
\hline & & Eleutherodactylus lamprotes & $\mathrm{CR}$ \\
\hline & & Eleutherodactylus nortoni & $\mathrm{CR}$ \\
\hline & & Eleutherodactylus oxyrhyncus & $\mathrm{CR}$ \\
\hline & & Eleutherodactylus parapelates & $\mathrm{CR}$ \\
\hline & & Eleutherodactylus paulsoni & CR \\
\hline & & Eleutherodactylus pictissimus & $\mathrm{VU}$ \\
\hline & & Eleutherodactylus ruthae & EN \\
\hline & & Eleutherodactylus sciagraphus & CR \\
\hline & & Eleutherodactylus semipalmatus & $\mathrm{CR}$ \\
\hline & & Eleutherodactylus thorectes & $\mathrm{CR}$ \\
\hline & & Eleutherodactylus ventrilineatus & $\mathrm{CR}$ \\
\hline & & Eleutherodactylus wetmorei & $\mathrm{VU}$ \\
\hline & & Hypsiboas heilprini & VU \\
\hline & & Lasiurus minor & VU \\
\hline & & Loxia megaplaga & EN \\
\hline & & Micropholis polita & VU \\
\hline & & Nectandra caudatoacuminata & $\mathrm{CR}$ \\
\hline & & Nectandra pulchra & $\mathrm{CR}$ \\
\hline & & Osteopilus pulchrilineatus & EN \\
\hline & & Osteopilus vastus & EN \\
\hline & & Plagiodontia aedium & EN \\
\hline & & Pouteria hotteana & EN \\
\hline & & Solenodon paradoxus & EN \\
\hline & & Tachycineta euchrysea & $\mathrm{VU}$ \\
\hline & & Xenoligea montana & VU \\
\hline & Massif de la Selle & Amazona ventralis & VU \\
\hline & & Aratinga chloroptera & VU \\
\hline & & Calyptophilus frugivorus & VU \\
\hline & & Catharus bicknelli & VU \\
\hline & & Cyclura cornuta & VU \\
\hline & & Eleutherodactylus alcoae & EN \\
\hline & & Eleutherodactylus armstrongi & EN \\
\hline & & Eleutherodactylus audanti & VU \\
\hline & & Eleutherodactylus darlingtoni & $\mathrm{CR}$ \\
\hline & & Eleutherodactylus fowleri & $\mathrm{CR}$ \\
\hline & & Eleutherodactylus furcyensis & $\mathrm{CR}$ \\
\hline & & Eleutherodactylus glanduliferoides & $\mathrm{CR}$ \\
\hline & & Eleutherodactylus heminota & EN \\
\hline & & Eleutherodactylus hypostenor & EN \\
\hline & & Eleutherodactylus jugans & $\mathrm{CR}$ \\
\hline & & Eleutherodactylus leoncei & $\mathrm{CR}$ \\
\hline & & Eleutherodactylus nortoni & CR \\
\hline & & Eleutherodactylus oxyrhyncus & $\mathrm{CR}$ \\
\hline & & Eleutherodactylus paulsoni & $\mathrm{CR}$ \\
\hline & & Eleutherodactylus pictissimus & VU \\
\hline & & Eleutherodactylus ruthae & EN \\
\hline & & Eleutherodactylus semipalmatus & $\mathrm{CR}$ \\
\hline
\end{tabular}




\begin{tabular}{|c|c|c|c|}
\hline Country & Key Biodiversity Area & Species scientific name & $\begin{array}{l}\text { IUCN Red List } \\
\text { Category }\end{array}$ \\
\hline & & Eleutherodactylus wetmorei & VU \\
\hline & & Hypsiboas heilprini & VU \\
\hline & & Loxia megaplaga & EN \\
\hline & & Mappia racemosa & VU \\
\hline & & Osteopilus pulchrilineatus & EN \\
\hline & & Osteopilus vastus & EN \\
\hline & & Peltophryne guentheri & VU \\
\hline & & Plagiodontia aedium & EN \\
\hline & & Pterodroma hasitata & EN \\
\hline & & Tachycineta euchrysea & VU \\
\hline & & Turdus swalesi & EN \\
\hline & & Xenoligea montana & VU \\
\hline & Morne Bailly & Eleutherodactylus poolei & $\mathrm{CR}$ \\
\hline & & Eleutherodactylus schmidti & CR \\
\hline & & Eleutherodactylus wetmorei & VU \\
\hline & & Hypsiboas heilprini & $\mathrm{VU}$ \\
\hline & & Osteopilus pulchrilineatus & EN \\
\hline & & Osteopilus vastus & EN \\
\hline & Pic Tete Boeuf & Eleutherodactylus glaphycompus & EN \\
\hline & & Eleutherodactylus heminota & EN \\
\hline & & Eleutherodactylus paulsoni & CR \\
\hline & & Eleutherodactylus pictissimus & VU \\
\hline & & Eleutherodactylus wetmorei & VU \\
\hline & & Hypsiboas heilprini & VU \\
\hline & Presqu'ile du Nord-Ouest I & Eleutherodactylus grahami & EN \\
\hline & & Eleutherodactylus Iucioi & CR \\
\hline & & Peltophryne guentheri & VU \\
\hline & Presqu'ile du Nord-Ouest II & Eleutherodactylus rhodesi & $\mathrm{CR}$ \\
\hline & & Peltophryne guentheri & VU \\
\hline & Trou Caïman & Amazona ventralis & VU \\
\hline & & Aratinga chloroptera & VU \\
\hline & & Corvus leucognaphalus & VU \\
\hline & & Dendrocygna arborea & VU \\
\hline & & Peltophryne guentheri & VU \\
\hline \multirow[t]{23}{*}{ Jamaica } & Black River Great Morass & Crocodylus acutus & VU \\
\hline & & Dendrocygna arborea & VU \\
\hline & & Eleutherodactylus luteolus & EN \\
\hline & & Eugenia brownei & VU \\
\hline & & Samyda glabrata & VU \\
\hline & Blue Mountains & Amazona collaria & VU \\
\hline & & Annona praetermissa & VU \\
\hline & & Ardisia brittonii & EN \\
\hline & & Bactris jamaicana & VU \\
\hline & & Brunfelsia jamaicensis & VU \\
\hline & & Bunchosia jamaicensis & $\mathrm{VU}$ \\
\hline & & Catharus bicknelli & VU \\
\hline & & Eleutherodactylus alticola & $\mathrm{CR}$ \\
\hline & & Eleutherodactylus andrewsi & EN \\
\hline & & Eleutherodactylus jamaicensis & EN \\
\hline & & Eleutherodactylus nubicola & EN \\
\hline & & Eleutherodactylus orcutti & $\mathrm{CR}$ \\
\hline & & Eleutherodactylus pentasyringos & VU \\
\hline & & Epicrates subflavus & VU \\
\hline & & Eugenia brachythrix & VU \\
\hline & & Eugenia crenata & VU \\
\hline & & Eugenia nicholsii & EN \\
\hline & & Eugenia rendlei & $\mathrm{CR}$ \\
\hline
\end{tabular}




\begin{tabular}{|c|c|c|c|}
\hline Country & Key Biodiversity Area & Species scientific name & $\begin{array}{l}\text { IUCN Red List } \\
\text { Category }\end{array}$ \\
\hline & & Geocapromys brownii & VU \\
\hline & & Hernandia catalpifolia & VU \\
\hline & & Hyeronima jamaicensis & VU \\
\hline & & Ilex jamaicana & EN \\
\hline & & Ilex puberula & $\mathrm{VU}$ \\
\hline & & Ilex puberula & VU \\
\hline & & Ilex puberula & VU \\
\hline & & Lunania polydactyla & $\mathrm{VU}$ \\
\hline & & Maytenus harrisii & $\mathrm{CR}$ \\
\hline & & Miconia nubicola & EN \\
\hline & & Nesopsar nigerrimus & EN \\
\hline & & Nowellia wrightii & VU \\
\hline & & Osteopilus wilderi & EN \\
\hline & & Patagioenas caribaea & VU \\
\hline & & Psychotria foetens & VU \\
\hline & & Rondeletia brachyphylla & EN \\
\hline & & Rondeletia hirsuta & VU \\
\hline & & Rondeletia hirta & VU \\
\hline & & Sebastiania alpina & VU \\
\hline & & Tachycineta euchrysea & $\mathrm{VU}$ \\
\hline & & Verbesina rupestris & VU \\
\hline & & Viburnum arboreum & VU \\
\hline & & Viburnum arboreum & VU \\
\hline & & Wallenia fawcettii & VU \\
\hline & & Zanthoxylum harrisii & VU \\
\hline & & Zanthoxylum hartii & VU \\
\hline & Bluefields & Amazona collaria & $\mathrm{VU}$ \\
\hline & & Epicrates subflavus & VU \\
\hline & & Eugenia abbreviata & EN \\
\hline & & Lasiurus degelidus & VU \\
\hline & Brazilleto Mountains & Eugenia eperforata & EN \\
\hline & & Geocapromys brownii & VU \\
\hline & & Malpighia proctorii & $\mathrm{CR}$ \\
\hline & Bull Bay & Brunfelsia membranacea & VU \\
\hline & & Bursera hollickii & EN \\
\hline & & Eleutherodactylus andrewsi & EN \\
\hline & & Eleutherodactylus orcutti & CR \\
\hline & & Guaiacum officinale & EN \\
\hline & & Lasiurus degelidus & VU \\
\hline & & Osteopilus wilderi & EN \\
\hline & & Phialanthus jamaicensis & EN \\
\hline & & Portlandia albiflora & $\mathrm{CR}$ \\
\hline & & Tetrasiphon jamaicensis & EN \\
\hline & Catadupa & Amazona agilis & $\mathrm{VU}$ \\
\hline & & Amazona collaria & $\mathrm{VU}$ \\
\hline & & Bactris jamaicana & VU \\
\hline & & Eleutherodactylus cundalli & VU \\
\hline & & Eleutherodactylus fuscus & $\mathrm{CR}$ \\
\hline & & Eleutherodactylus grabhami & EN \\
\hline & & Eleutherodactylus griphus & $\mathrm{CR}$ \\
\hline & & Eleutherodactylus jamaicensis & EN \\
\hline & & Eleutherodactylus luteolus & EN \\
\hline & & Guarea jamaicensis & VU \\
\hline & & Guettarda longiflora & $\mathrm{CR}$ \\
\hline & & Malpighia obtusifolia & VU \\
\hline & & Osteopilus crucialis & EN \\
\hline & & Osteopilus marianae & EN \\
\hline
\end{tabular}




\begin{tabular}{|c|c|c|c|}
\hline Country & Key Biodiversity Area & Species scientific name & $\begin{array}{l}\text { IUCN Red List } \\
\text { Category }\end{array}$ \\
\hline & & Osteopilus wilderi & EN \\
\hline & & Ouratea elegans & $\mathrm{CR}$ \\
\hline & & Patagioenas caribaea & VU \\
\hline & & Phyllanthus cauliflorus & VU \\
\hline & & Pimenta obscura & $\mathrm{VU}$ \\
\hline & & Ternstroemia glomerata & CR \\
\hline & Caymanas & Eleutherodactylus jamaicensis & EN \\
\hline & & Geocapromys brownii & VU \\
\hline & Cockpit Country & Alvaradoa jamaicensis & VU \\
\hline & & Amazona agilis & VU \\
\hline & & Amazona collaria & VU \\
\hline & & Brunfelsia splendida & VU \\
\hline & & Bursera aromatica & VU \\
\hline & & Buxus arborea & VU \\
\hline & & Calliandra comosa & VU \\
\hline & & Calyptranthes nodosa & $\mathrm{VU}$ \\
\hline & & Cassipourea brittoniana & EN \\
\hline & & Cassipourea subcordata & CR \\
\hline & & Coccoloba troyana & VU \\
\hline & & Colubrina obscura & $\mathrm{VU}$ \\
\hline & & Comocladia cordata & VU \\
\hline & & Cordia harrisii & VU \\
\hline & & Eleutherodactylus cundalli & VU \\
\hline & & Eleutherodactylus fuscus & $\mathrm{CR}$ \\
\hline & & Eleutherodactylus grabhami & EN \\
\hline & & Eleutherodactylus griphus & $\mathrm{CR}$ \\
\hline & & Eleutherodactylus jamaicensis & EN \\
\hline & & Eleutherodactylus junori & $\mathrm{CR}$ \\
\hline & & Eleutherodactylus luteolus & EN \\
\hline & & Eleutherodactylus sisyphodemus & CR \\
\hline & & Epicrates subflavus & VU \\
\hline & & Erithalis quadrangularis & VU \\
\hline & & Eugenia laurae & EN \\
\hline & & Eugenia sachetae & EN \\
\hline & & Eugenia schulziana & VU \\
\hline & & Exostema orbiculatum & $\mathrm{CR}$ \\
\hline & & Geocapromys brownii & VU \\
\hline & & Guettarda longiflora & $\mathrm{CR}$ \\
\hline & & Hamelia papillosa & VU \\
\hline & & Lasiocroton trelawniensis & EN \\
\hline & & Lunania polydactyla & VU \\
\hline & & Malpighia obtusifolia & VU \\
\hline & & Manilkara excisa & EN \\
\hline & & Mitranthes macrophylla & CR \\
\hline & & Nesopsar nigerrimus & EN \\
\hline & & Osteopilus crucialis & EN \\
\hline & & Osteopilus marianae & EN \\
\hline & & Osteopilus wilderi & EN \\
\hline & & Patagioenas caribaea & $\mathrm{VU}$ \\
\hline & & Phyllanthus axillaris & EN \\
\hline & & Pimenta obscura & $\mathrm{VU}$ \\
\hline & & Pimenta richardii & EN \\
\hline & & Podocarpus purdieanus & EN \\
\hline & & Psychotria plicata & VU \\
\hline & & Psychotria siphonophora & EN \\
\hline & & Rochefortia acrantha & $\mathrm{VU}$ \\
\hline & & Rochefortia acrantha & VU \\
\hline
\end{tabular}




\begin{tabular}{|c|c|c|c|}
\hline Country & Key Biodiversity Area & Species scientific name & $\begin{array}{l}\text { IUCN Red List } \\
\text { Category }\end{array}$ \\
\hline & & Rondeletia amplexicaulis & EN \\
\hline & & Schoepfia harrisii & VU \\
\hline & & Scolosanthus howardii & EN \\
\hline & & Scolosanthus howardii & EN \\
\hline & & Sophora saxicola & EN \\
\hline & & Spathelia coccinea & $\mathrm{CR}$ \\
\hline & & Strempeliopsis arborea & VU \\
\hline & & Tachycineta euchrysea & $\mathrm{VU}$ \\
\hline & & Trachemys terrapen & VU \\
\hline & & Viburnum arboreum & VU \\
\hline & & Xylosma proctorii & VU \\
\hline & Dolphin Head & Calyptranthes acutissima & $\mathrm{CR}$ \\
\hline & & Calyptranthes acutissima & $\mathrm{CR}$ \\
\hline & & Calyptranthes discolor & EN \\
\hline & & Cassipourea subsessilis & CR \\
\hline & & Comocladia cordata & VU \\
\hline & & Comocladia parvifoliola & $\mathrm{CR}$ \\
\hline & & Dendropanax cordifolius & CR \\
\hline & & Eleutherodactylus cundalli & VU \\
\hline & & Eleutherodactylus fuscus & $\mathrm{CR}$ \\
\hline & & Eleutherodactylus grabhami & EN \\
\hline & & Eleutherodactylus jamaicensis & EN \\
\hline & & Eleutherodactylus Iuteolus & EN \\
\hline & & Eugenia polypora & $\mathrm{CR}$ \\
\hline & & Lasiocroton fawcettii & VU \\
\hline & & Ormosia jamaicensis & EN \\
\hline & & Osteopilus crucialis & EN \\
\hline & & Osteopilus wilderi & EN \\
\hline & & Patagioenas caribaea & VU \\
\hline & & Psychotria hanoverensis & CR \\
\hline & & Rondeletia cincta & $\mathrm{CR}$ \\
\hline & & Sebastiania fasciculata & EN \\
\hline & & Tabernaemontana ochroleuca & VU \\
\hline & & Tabernaemontana ovalifolia & EN \\
\hline & Don Figuerero Mountains & Coccoloba proctorii & EN \\
\hline & & Colubrina obscura & VU \\
\hline & & Eleutherodactylus cundalli & VU \\
\hline & & Eleutherodactylus grabhami & EN \\
\hline & & Eleutherodactylus jamaicensis & EN \\
\hline & & Erythroxylum incrassatum & VU \\
\hline & & Osteopilus crucialis & EN \\
\hline & & Osteopilus marianae & EN \\
\hline & & Osteopilus wilderi & EN \\
\hline & Font Hill & Crocodylus acutus & $\mathrm{VU}$ \\
\hline & Great River & Eleutherodactylus cundalli & $\mathrm{VU}$ \\
\hline & & Eleutherodactylus fuscus & $\mathrm{CR}$ \\
\hline & & Eleutherodactylus jamaicensis & EN \\
\hline & & Eleutherodactylus luteolus & EN \\
\hline & & Geocapromys brownii & $\mathrm{VU}$ \\
\hline & & Osteopilus crucialis & EN \\
\hline & & Osteopilus wilderi & EN \\
\hline & Hellshire Hills & Bursera hollickii & EN \\
\hline & & Cyclura collei & $\mathrm{CR}$ \\
\hline & & Dendrocygna arborea & VU \\
\hline & & Epicrates subflavus & VU \\
\hline & & Geocapromys brownii & $\mathrm{VU}$ \\
\hline & & Guaiacum officinale & EN \\
\hline
\end{tabular}




\begin{tabular}{|c|c|c|c|}
\hline Country & Key Biodiversity Area & Species scientific name & $\begin{array}{l}\text { IUCN Red List } \\
\text { Category }\end{array}$ \\
\hline & & Lunania polydactyla & VU \\
\hline & & Phialanthus revolutus & EN \\
\hline & & Siphonorhis americana & $\mathrm{CR}$ \\
\hline & & Zanthoxylum harrisii & VU \\
\hline & John Crow Mountains & Amazona agilis & $\mathrm{VU}$ \\
\hline & & Amazona collaria & VU \\
\hline & & Bactris jamaicana & VU \\
\hline & & Clusia portlandiana & $\mathrm{VU}$ \\
\hline & & Dendropanax blakeanus & VU \\
\hline & & Eleutherodactylus andrewsi & EN \\
\hline & & Eleutherodactylus jamaicensis & EN \\
\hline & & Eleutherodactylus orcutti & $\mathrm{CR}$ \\
\hline & & Eleutherodactylus pentasyringos & VU \\
\hline & & Eugenia rendlei & $\mathrm{CR}$ \\
\hline & & Geocapromys brownii & VU \\
\hline & & Ilex jamaicana & EN \\
\hline & & Ilex jamaicana & EN \\
\hline & & Myrcia calcicola & VU \\
\hline & & Nesopsar nigerrimus & EN \\
\hline & & Osteopilus wilderi & EN \\
\hline & & Patagioenas caribaea & VU \\
\hline & & Phyllanthus eximius & VU \\
\hline & & Psychotria bryonicola & $\mathrm{CR}$ \\
\hline & & Psychotria clusioides & EN \\
\hline & & Pterodroma caribbaea & $\mathrm{CR}$ \\
\hline & & Rondeletia portlandensis & VU \\
\hline & & Sideroxylon bullatum & VU \\
\hline & & Ternstroemia howardiana & VU \\
\hline & & Wallenia sylvestris & VU \\
\hline & & Weinmannia portlandiana & VU \\
\hline & Johnson Mountain Hills & Bactris jamaicana & VU \\
\hline & Kellets Camperdown Area & Eleutherodactylus cundalli & VU \\
\hline & & Eleutherodactylus grabhami & EN \\
\hline & & Eleutherodactylus jamaicensis & EN \\
\hline & & Eleutherodactylus junori & $\mathrm{CR}$ \\
\hline & & Geocapromys brownii & VU \\
\hline & & Osteopilus crucialis & EN \\
\hline & & Osteopilus marianae & EN \\
\hline & & Osteopilus wilderi & EN \\
\hline & Litchfield Mountain - Matheson's Run & Acidocroton verrucosus & VU \\
\hline & & Amazona agilis & VU \\
\hline & & Amazona collaria & VU \\
\hline & & Ardisia byrsonimae & $\mathrm{CR}$ \\
\hline & & Calyptranthes capitata & VU \\
\hline & & Carica jamaicensis & $\mathrm{VU}$ \\
\hline & & Clusia clarendonensis & VU \\
\hline & & Coccoloba troyana & VU \\
\hline & & Colubrina obscura & VU \\
\hline & & Cordia clarendonensis & $\mathrm{VU}$ \\
\hline & & Dendropanax filipes & $\mathrm{CR}$ \\
\hline & & Dendropanax grandiflorus & $\mathrm{CR}$ \\
\hline & & Eleutherodactylus cundalli & VU \\
\hline & & Eleutherodactylus grabhami & EN \\
\hline & & Eleutherodactylus jamaicensis & EN \\
\hline & & Erithalis quadrangularis & VU \\
\hline & & Erythroxylum jamaicense & $\mathrm{VU}$ \\
\hline & & Eugenia aboukirensis & $\mathrm{CR}$ \\
\hline
\end{tabular}




\begin{tabular}{|c|c|c|c|}
\hline Country & Key Biodiversity Area & Species scientific name & $\begin{array}{l}\text { IUCN Red List } \\
\text { Category }\end{array}$ \\
\hline & & Eugenia eperforata & EN \\
\hline & & Eugenia heterochroa & VU \\
\hline & & Eugenia lamprophylla & VU \\
\hline & & Eugenia schulziana & VU \\
\hline & & Geocapromys brownii & $\mathrm{VU}$ \\
\hline & & Guarea jamaicensis & VU \\
\hline & & Lasiocroton harrisii & VU \\
\hline & & Malpighia harrisii & $\mathrm{VU}$ \\
\hline & & Mitranthes nivea & EN \\
\hline & & Nesopsar nigerrimus & EN \\
\hline & & Ocotea staminoides & EN \\
\hline & & Osteopilus crucialis & EN \\
\hline & & Osteopilus marianae & EN \\
\hline & & Osteopilus wilderi & EN \\
\hline & & Palicourea wilesii & VU \\
\hline & & Patagioenas caribaea & $\mathrm{VU}$ \\
\hline & & Portlandia harrisii & VU \\
\hline & & Psychotria clarendonensis & EN \\
\hline & & Rondeletia adamsii & VU \\
\hline & & Rondeletia clarendonensis & EN \\
\hline & & Samyda glabrata & VU \\
\hline & & Schefflera troyana & VU \\
\hline & & Schoepfia harrisii & VU \\
\hline & & Sebastiania spicata & EN \\
\hline & & Ternstroemia bullata & $\mathrm{CR}$ \\
\hline & & Ternstroemia calycina & EN \\
\hline & Main Ridge & Eleutherodactylus cundalli & $\mathrm{VU}$ \\
\hline & & Eleutherodactylus grabhami & EN \\
\hline & & Eleutherodactylus jamaicensis & EN \\
\hline & & Eleutherodactylus junori & CR \\
\hline & & Geocapromys brownii & VU \\
\hline & & Osteopilus crucialis & EN \\
\hline & & Osteopilus marianae & EN \\
\hline & & Osteopilus wilderi & EN \\
\hline & May Pen & Eleutherodactylus cundalli & VU \\
\hline & & Eleutherodactylus jamaicensis & EN \\
\hline & & Lasiurus degelidus & VU \\
\hline & & Osteopilus crucialis & EN \\
\hline & & Osteopilus wilderi & EN \\
\hline & Milk River & Trachemys terrapen & VU \\
\hline & Mocho Mountains & Eleutherodactylus cundalli & VU \\
\hline & & Eleutherodactylus grabhami & EN \\
\hline & & Eleutherodactylus jamaicensis & EN \\
\hline & & Osteopilus crucialis & EN \\
\hline & & Osteopilus wilderi & EN \\
\hline & Mount Diablo & Alvaradoa jamaicensis & VU \\
\hline & & Amazona agilis & VU \\
\hline & & Amazona collaria & VU \\
\hline & & Brunfelsia splendida & $\mathrm{VU}$ \\
\hline & & Carica jamaicensis & VU \\
\hline & & Eleutherodactylus cundalli & $\mathrm{VU}$ \\
\hline & & Eleutherodactylus grabhami & EN \\
\hline & & Eleutherodactylus jamaicensis & EN \\
\hline & & Eleutherodactylus junori & $\mathrm{CR}$ \\
\hline & & Erythroxylum incrassatum & VU \\
\hline & & Eugenia acutisepala & EN \\
\hline & & Exostema triflorum & VU \\
\hline
\end{tabular}




\begin{tabular}{|c|c|c|c|}
\hline Country & Key Biodiversity Area & Species scientific name & $\begin{array}{l}\text { IUCN Red List } \\
\text { Category }\end{array}$ \\
\hline & & Exostema triflorum & VU \\
\hline & & Geocapromys brownii & VU \\
\hline & & Gymnanthes glandulosa & VU \\
\hline & & Ilex florifera & VU \\
\hline & & Ilex subtriflora & $\mathrm{CR}$ \\
\hline & & Malpighia cauliflora & EN \\
\hline & & Malpighia harrisii & VU \\
\hline & & Nesopsar nigerrimus & EN \\
\hline & & Osteopilus crucialis & EN \\
\hline & & Osteopilus marianae & EN \\
\hline & & Osteopilus wilderi & EN \\
\hline & & Palicourea wilesii & VU \\
\hline & & Podocarpus purdieanus & EN \\
\hline & & Trachemys terrapen & VU \\
\hline & Negril & Crocodylus acutus & VU \\
\hline & & Dendrocygna arborea & $\mathrm{VU}$ \\
\hline & & Eleutherodactylus cundalli & VU \\
\hline & & Eleutherodactylus luteolus & EN \\
\hline & & Epicrates subflavus & VU \\
\hline & & Erithalis quadrangularis & VU \\
\hline & & Trachemys terrapen & VU \\
\hline & & Zamia amblyphyllidia & VU \\
\hline & & Zanthoxylum negrilense & EN \\
\hline & North Coast Forest & Amazona collaria & VU \\
\hline & & Eleutherodactylus cundalli & VU \\
\hline & & Epicrates subflavus & VU \\
\hline & & Epicrates subflavus & $\mathrm{VU}$ \\
\hline & & Lasiurus degelidus & VU \\
\hline & & Osteopilus wilderi & EN \\
\hline & & Patagioenas caribaea & VU \\
\hline & & Trachemys terrapen & VU \\
\hline & Peckam Woods & Acidocroton verrucosus & VU \\
\hline & & Ardisia byrsonimae & $\mathrm{CR}$ \\
\hline & & Clusia clarendonensis & VU \\
\hline & & Coccoloba troyana & VU \\
\hline & & Cordia clarendonensis & VU \\
\hline & & Dendropanax filipes & CR \\
\hline & & Dendropanax grandiflorus & $\mathrm{CR}$ \\
\hline & & Erythroxylum jamaicense & VU \\
\hline & & Eugenia heterochroa & VU \\
\hline & & Eugenia lamprophylla & VU \\
\hline & & Lasiocroton harrisii & VU \\
\hline & & Lasiocroton trelawniensis & EN \\
\hline & & Malpighia harrisii & VU \\
\hline & & Palicourea wilesii & $\mathrm{VU}$ \\
\hline & & Portlandia harrisii & VU \\
\hline & & Psychotria clarendonensis & EN \\
\hline & & Rondeletia adamsii & VU \\
\hline & & Rondeletia clarendonensis & EN \\
\hline & & Samyda glabrata & VU \\
\hline & & Schefflera troyana & VU \\
\hline & & Schoepfia harrisii & VU \\
\hline & & Sebastiania spicata & EN \\
\hline & & Ternstroemia bullata & $\mathrm{CR}$ \\
\hline & & Ternstroemia calycina & EN \\
\hline & Point Hill & Eleutherodactylus cundalli & $\mathrm{VU}$ \\
\hline & & Eleutherodactylus jamaicensis & EN \\
\hline
\end{tabular}




\begin{tabular}{|c|c|c|c|}
\hline Country & Key Biodiversity Area & Species scientific name & $\begin{array}{l}\text { IUCN Red List } \\
\text { Category }\end{array}$ \\
\hline & & Geocapromys brownii & VU \\
\hline & & Natalus jamaicensis & $\mathrm{CR}$ \\
\hline & & Osteopilus crucialis & EN \\
\hline & & Osteopilus wilderi & EN \\
\hline & Portland Ridge and Bight & Crocodylus acutus & $\mathrm{VU}$ \\
\hline & & Dendrocygna arborea & VU \\
\hline & & Eleutherodactylus cavernicola & $\mathrm{CR}$ \\
\hline & & Epicrates subflavus & VU \\
\hline & & Eugenia eperforata & EN \\
\hline & & Malpighia proctorii & $\mathrm{CR}$ \\
\hline & & Natalus jamaicensis & $\mathrm{CR}$ \\
\hline & Red Ground & Geocapromys brownii & VU \\
\hline & & Osteopilus crucialis & EN \\
\hline & Rio Cobre & Eleutherodactylus jamaicensis & EN \\
\hline & & Geocapromys brownii & VU \\
\hline & & Osteopilus crucialis & EN \\
\hline & & Trachemys terrapen & VU \\
\hline & Rio Grande & Eleutherodactylus jamaicensis & EN \\
\hline & Rio Grande & Eleutherodactylus pentasyringos & VU \\
\hline & & Osteopilus wilderi & EN \\
\hline & & Trachemys terrapen & VU \\
\hline & Rio Magno & Eleutherodactylus jamaicensis & EN \\
\hline & & Geocapromys brownii & VU \\
\hline & & Osteopilus wilderi & EN \\
\hline & Rio Pedro & Eleutherodactylus jamaicensis & EN \\
\hline & & Geocapromys brownii & VU \\
\hline & & Osteopilus crucialis & EN \\
\hline & & Osteopilus wilderi & EN \\
\hline & Santa Cruz Mountains & Eleutherodactylus cundalli & VU \\
\hline & & Geocapromys brownii & VU \\
\hline & & Osteopilus wilderi & EN \\
\hline & & Rhamnidium dictyophyllum & EN \\
\hline & Stephney Johns Vale-Bull Head & Carica jamaicensis & VU \\
\hline & & Colubrina obscura & VU \\
\hline & & Eleutherodactylus cundalli & VU \\
\hline & & Eleutherodactylus grabhami & EN \\
\hline & & Eleutherodactylus jamaicensis & EN \\
\hline & & Eleutherodactylus junori & $\mathrm{CR}$ \\
\hline & & Erythroxylum jamaicense & VU \\
\hline & & Geocapromys brownii & VU \\
\hline & & Malpighia harrisii & VU \\
\hline & & Mitranthes nivea & EN \\
\hline & & Osteopilus crucialis & EN \\
\hline & & Osteopilus marianae & EN \\
\hline & & Osteopilus wilderi & EN \\
\hline & Swift River & Trachemys terrapen & VU \\
\hline & Wag Water River & Osteopilus wilderi & EN \\
\hline & & Trachemys terrapen & VU \\
\hline & White Rock Hill & Guarea jamaicensis & $\mathrm{VU}$ \\
\hline & & Pimenta obscura & VU \\
\hline & & Ternstroemia glomerata & $\mathrm{CR}$ \\
\hline & Yallahs & Epicrates subflavus & VU \\
\hline & & Patagioenas caribaea & VU \\
\hline \multirow[t]{4}{*}{ Martinique } & Forêts du Nord et de la Montagne Pelée & Allobates chalcopis & VU \\
\hline & & Icterus bonana & VU \\
\hline & & Iguana delicatissima & $\mathrm{VU}$ \\
\hline & & Myotis martiniquensis & VU \\
\hline
\end{tabular}




\begin{tabular}{|c|c|c|c|}
\hline Country & Key Biodiversity Area & Species scientific name & $\begin{array}{l}\text { IUCN Red List } \\
\text { Category }\end{array}$ \\
\hline & Grand Macabou & Icterus bonana & VU \\
\hline & Ilets Boiseau et Petit Piton & Iguana delicatissima & VU \\
\hline & Mangrove de Fort de France & Icterus bonana & VU \\
\hline & Massif forestier entre Le Diamant et les Trois-îlets & Icterus bonana & VU \\
\hline & Pitons du Carbet & Freziera cordata & $\mathrm{VU}$ \\
\hline & & Icterus bonana & VU \\
\hline & & Iguana delicatissima & VU \\
\hline & & Inga martinicensis & $\mathrm{VU}$ \\
\hline & & Myotis martiniquensis & VU \\
\hline & & Pouteria pallida & EN \\
\hline & & Pouteria semecarpifolia & VU \\
\hline & & Schefflera urbaniana & VU \\
\hline & Presqu'île de la Caravelle & Icterus bonana & VU \\
\hline & & Myotis martiniquensis & VU \\
\hline & & Ramphocinclus brachyurus & EN \\
\hline & Rocher du Diamant & Liophis cursor & $\mathrm{CR}$ \\
\hline \multirow[t]{12}{*}{ Montserrat } & Centre Hills & Cedrela odorata & VU \\
\hline & & Chiroderma improvisum & VU \\
\hline & & Diploglossus montisserrati & $\mathrm{CR}$ \\
\hline & & Guaiacum officinale & EN \\
\hline & & Icterus oberi & $\mathrm{CR}$ \\
\hline & & Leptodactylus fallax & $\mathrm{CR}$ \\
\hline & & Sturnira thomasi & VU \\
\hline & & Turdus Iherminieri & VU \\
\hline & Northern Forested Ghauts & Leptodactylus fallax & $\mathrm{CR}$ \\
\hline & & Turdus Iherminieri & VU \\
\hline & South Soufriere Hills & Icterus oberi & $\mathrm{CR}$ \\
\hline & & Turdus Iherminieri & VU \\
\hline \multirow[t]{29}{*}{ Puerto Rico } & Acantilados del Noroeste & Antirhea portoricensis & VU \\
\hline & & Auerodendron pauciflorum & CR \\
\hline & & Erythrina eggersii & EN \\
\hline & & Goetzea elegans & EN \\
\hline & & Manilkara pleeana & VU \\
\hline & & Manilkara valenzuelana & VU \\
\hline & & Picrasma excelsa & VU \\
\hline & & Schoepfia arenaria & EN \\
\hline & & Zamia amblyphyllidia & VU \\
\hline & & Zanthoxylum flavum & VU \\
\hline & Bahía de Jobos & Agelaius xanthomus & EN \\
\hline & Baños de Coamo & Chrysophyllum pauciflorum & VU \\
\hline & & Erythrina eggersii & EN \\
\hline & & Guaiacum officinale & EN \\
\hline & & Leptocereus quadricostatus & $\mathrm{CR}$ \\
\hline & & Manilkara pleeana & VU \\
\hline & & Peltophryne lemur & $\mathrm{CR}$ \\
\hline & & Picrasma excelsa & VU \\
\hline & & Solanum drymophilum & $\mathrm{CR}$ \\
\hline & & Stahlia monosperma & EN \\
\hline & Bosque de Vega & Erythrina eggersii & EN \\
\hline & & Stenoderma rufum & VU \\
\hline & Caño Tiburones & Dendrocygna arborea & VU \\
\hline & & Erythrina eggersii & EN \\
\hline & & Manilkara pleeana & VU \\
\hline & Carite & Eleutherodactylus eneidae & $\mathrm{CR}$ \\
\hline & & Eleutherodactylus gryllus & EN \\
\hline & & Eleutherodactylus hedricki & EN \\
\hline & & Eleutherodactylus jasperi & $\mathrm{CR}$ \\
\hline
\end{tabular}




\begin{tabular}{|c|c|c|c|}
\hline Country & Key Biodiversity Area & Species scientific name & $\begin{array}{l}\text { IUCN Red List } \\
\text { Category }\end{array}$ \\
\hline & & Eleutherodactylus karlschmidti & $\mathrm{CR}$ \\
\hline & & Eleutherodactylus locustus & $\mathrm{CR}$ \\
\hline & & Eleutherodactylus portoricensis & EN \\
\hline & & Eleutherodactylus richmondi & $\mathrm{CR}$ \\
\hline & & Eleutherodactylus wightmanae & EN \\
\hline & & Gaussia attenuata & VU \\
\hline & & Stenoderma rufum & VU \\
\hline & Ceiba y Naguabo & Chrysophyllum pauciflorum & $\mathrm{VU}$ \\
\hline & & Chrysophyllum pauciflorum & VU \\
\hline & & Coccoloba rugosa & EN \\
\hline & & Dermochelys coriacea & $\mathrm{CR}$ \\
\hline & & Guaiacum officinale & EN \\
\hline & & Maytenus cymosa & EN \\
\hline & & Schoepfia arenaria & EN \\
\hline & & Stahlia monosperma & EN \\
\hline & Ciénaga Las Cucharillas & Banara vanderbiltii & $\mathrm{CR}$ \\
\hline & & Buxus vahlii & $\mathrm{CR}$ \\
\hline & & Coccoloba rugosa & EN \\
\hline & & Dendrocygna arborea & VU \\
\hline & & Gaussia attenuata & VU \\
\hline & & Tabernaemontana oppositifolia & VU \\
\hline & Cordillera Central & Antirhea sintenisii & VU \\
\hline & & Callicarpa ampla & CR \\
\hline & & Chrysophyllum pauciflorum & VU \\
\hline & & Cornutia obovata & $\mathrm{CR}$ \\
\hline & & Eleutherodactylus eneidae & $\mathrm{CR}$ \\
\hline & & Eleutherodactylus gryllus & EN \\
\hline & & Eleutherodactylus hedricki & EN \\
\hline & & Eleutherodactylus portoricensis & EN \\
\hline & & Eleutherodactylus richmondi & CR \\
\hline & & Eleutherodactylus wightmanae & EN \\
\hline & & Gaussia attenuata & VU \\
\hline & & Ilex cookii & $\mathrm{CR}$ \\
\hline & & Juglans jamaicensis & VU \\
\hline & & Marlierea sintenisii & VU \\
\hline & & Nectandra krugii & EN \\
\hline & & Pouteria hotteana & EN \\
\hline & & Schefflera gleasonii & VU \\
\hline & & Stenoderma rufum & VU \\
\hline & Corredor Ecológico del Noreste & Coccoloba rugosa & EN \\
\hline & & Dermochelys coriacea & CR \\
\hline & & Goetzea elegans & EN \\
\hline & & Manilkara pleeana & VU \\
\hline & & Schoepfia arenaria & EN \\
\hline & & Stahlia monosperma & EN \\
\hline & Culebra & Anolis roosevelti & $\mathrm{CR}$ \\
\hline & & Guaiacum officinale & EN \\
\hline & & Maytenus cymosa & EN \\
\hline & El Yunque & Amazona vittata & $\mathrm{CR}$ \\
\hline & & Antirhea sintenisii & VU \\
\hline & & Dendroica angelae & VU \\
\hline & & Eleutherodactylus eneidae & CR \\
\hline & & Eleutherodactylus gryllus & EN \\
\hline & & Eleutherodactylus hedricki & EN \\
\hline & & Eleutherodactylus karlschmidti & CR \\
\hline & & Eleutherodactylus locustus & $\mathrm{CR}$ \\
\hline & & Eleutherodactylus portoricensis & EN \\
\hline
\end{tabular}




\begin{tabular}{|c|c|c|c|}
\hline Country & Key Biodiversity Area & Species scientific name & $\begin{array}{l}\text { IUCN Red List } \\
\text { Category }\end{array}$ \\
\hline & & Eleutherodactylus richmondi & $\mathrm{CR}$ \\
\hline & & Eleutherodactylus unicolor & VU \\
\hline & & Eleutherodactylus wightmanae & EN \\
\hline & & Lasiurus minor & VU \\
\hline & & Stenoderma rufum & $\mathrm{VU}$ \\
\hline & & Styrax portoricensis & CR \\
\hline & & Ternstroemia subsessilis & $\mathrm{CR}$ \\
\hline & Guaniquilla y Boquerón & Agelaius xanthomus & EN \\
\hline & & Eugenia woodburyana & $\mathrm{CR}$ \\
\hline & & Gaussia attenuata & VU \\
\hline & & Guaiacum officinale & EN \\
\hline & & Leptocereus quadricostatus & $\mathrm{CR}$ \\
\hline & Humacao & Coccoloba rugosa & EN \\
\hline & & Dendrocygna arborea & VU \\
\hline & & Eretmochelys imbricata & CR \\
\hline & Karso del Norte & Amazona vittata & $\mathrm{CR}$ \\
\hline & & Antirhea portoricensis & VU \\
\hline & & Antirhea sintenisii & VU \\
\hline & & Auerodendron pauciflorum & $\mathrm{CR}$ \\
\hline & & Banara vanderbiltii & $\mathrm{CR}$ \\
\hline & & Buxus vahlii & $\mathrm{CR}$ \\
\hline & & Chrysophyllum pauciflorum & VU \\
\hline & & Coccoloba rugosa & EN \\
\hline & & Cornutia obovata & $\mathrm{CR}$ \\
\hline & & Eleutherodactylus richmondi & $\mathrm{CR}$ \\
\hline & & Gaussia attenuata & VU \\
\hline & & Goetzea elegans & EN \\
\hline & & Goetzea elegans & EN \\
\hline & & Goetzea elegans & EN \\
\hline & & Henriettea membranifolia & CR \\
\hline & & Lasiurus minor & VU \\
\hline & & Manilkara pleeana & VU \\
\hline & & Mappia racemosa & VU \\
\hline & & Maytenus ponceana & VU \\
\hline & & Myrcia paganii & $\mathrm{CR}$ \\
\hline & & Nectandra krugii & EN \\
\hline & & Peltophryne lemur & CR \\
\hline & & Pleodendron macranthum & $\mathrm{CR}$ \\
\hline & & Schoepfia arenaria & EN \\
\hline & & Solanum drymophilum & $\mathrm{CR}$ \\
\hline & & Stenoderma rufum & VU \\
\hline & & Zamia amblyphyllidia & VU \\
\hline & Karso del Sur & Buxus vahlii & $\mathrm{CR}$ \\
\hline & & Caprimulgus noctitherus & $\mathrm{CR}$ \\
\hline & & Cordia rupicola & $\mathrm{CR}$ \\
\hline & & Eugenia woodburyana & $\mathrm{CR}$ \\
\hline & & Guaiacum officinale & EN \\
\hline & & Peltophryne lemur & $\mathrm{CR}$ \\
\hline & & Stahlia monosperma & EN \\
\hline & & Zamia portoricensis & $\mathrm{CR}$ \\
\hline & Laguna Tortuguero & Erythrina eggersii & EN \\
\hline & & Schoepfia arenaria & EN \\
\hline & Las Piedras Chiquitas & Chrysophyllum pauciflorum & VU \\
\hline & & Maytenus ponceana & VU \\
\hline & & Picrasma excelsa & VU \\
\hline & & Solanum drymophilum & $\mathrm{CR}$ \\
\hline & & Zanthoxylum thomasianum & EN \\
\hline
\end{tabular}




\begin{tabular}{|c|c|c|c|}
\hline Country & Key Biodiversity Area & Species scientific name & $\begin{array}{l}\text { IUCN Red List } \\
\text { Category }\end{array}$ \\
\hline & Maricao y Susúa & Antirhea portoricensis & VU \\
\hline & & Calyptranthes portoricensis & EN \\
\hline & & Caprimulgus noctitherus & $\mathrm{CR}$ \\
\hline & & Chrysophyllum pauciflorum & VU \\
\hline & & Chrysophyllum pauciflorum & $\mathrm{VU}$ \\
\hline & & Coccoloba rugosa & EN \\
\hline & & Cornutia obovata & $\mathrm{CR}$ \\
\hline & & Dendroica angelae & $\mathrm{VU}$ \\
\hline & & Eleutherodactylus eneidae & $\mathrm{CR}$ \\
\hline & & Eleutherodactylus gryllus & EN \\
\hline & & Eleutherodactylus portoricensis & EN \\
\hline & & Eleutherodactylus richmondi & $\mathrm{CR}$ \\
\hline & & Eleutherodactylus wightmanae & EN \\
\hline & & Erythrina eggersii & EN \\
\hline & & Gaussia attenuata & VU \\
\hline & & Lasiurus minor & $\mathrm{VU}$ \\
\hline & & Maytenus ponceana & VU \\
\hline & & Pouteria hotteana & EN \\
\hline & & Pouteria hotteana & EN \\
\hline & & Schefflera gleasonii & $\mathrm{VU}$ \\
\hline & & Schoepfia arenaria & EN \\
\hline & & Stahlia monosperma & EN \\
\hline & & Stenoderma rufum & VU \\
\hline & & Tabernaemontana oppositifolia & VU \\
\hline & & Xylosma pachyphyllum & $\mathrm{CR}$ \\
\hline & Mona y Monito & Agelaius xanthomus & EN \\
\hline & & Cyclura cornuta & $\mathrm{VU}$ \\
\hline & & Eleutherodactylus monensis & VU \\
\hline & & Eretmochelys imbricata & $\mathrm{CR}$ \\
\hline & & Guaiacum sanctum & EN \\
\hline & & Sphaerodactylus micropithecus & EN \\
\hline & & Typhlops monensis & EN \\
\hline & Piñones & Dermochelys coriacea & $\mathrm{CR}$ \\
\hline & & Erythrina eggersii & EN \\
\hline & & Schoepfia arenaria & EN \\
\hline & Sabana Seca & Eleutherodactylus juanariveroi & $\mathrm{CR}$ \\
\hline & Salinas de Punta Cucharas & Cordia rupicola & CR \\
\hline & & Erythrina eggersii & EN \\
\hline & & Guaiacum officinale & EN \\
\hline & & Guaiacum sanctum & EN \\
\hline & & Leptocereus quadricostatus & $\mathrm{CR}$ \\
\hline & & Maytenus ponceana & VU \\
\hline & & Trichilia triacantha & $\mathrm{CR}$ \\
\hline & Sierra Bermeja y Laguna Cartagena & Agelaius xanthomus & EN \\
\hline & & Caprimulgus noctitherus & $\mathrm{CR}$ \\
\hline & & Dendrocygna arborea & VU \\
\hline & & Eugenia woodburyana & $\mathrm{CR}$ \\
\hline & & Manilkara pleeana & VU \\
\hline & & Stahlia monosperma & EN \\
\hline & Sierra de Pandura & Eleutherodactylus cooki & VU \\
\hline & & Eleutherodactylus locustus & $\mathrm{CR}$ \\
\hline & & Eleutherodactylus portoricensis & EN \\
\hline & Suroeste & Agelaius xanthomus & EN \\
\hline & & Caprimulgus noctitherus & $\mathrm{CR}$ \\
\hline & & Eugenia woodburyana & $\mathrm{CR}$ \\
\hline & & Guaiacum officinale & EN \\
\hline & & Leptocereus quadricostatus & $\mathrm{CR}$ \\
\hline
\end{tabular}




\begin{tabular}{|c|c|c|c|}
\hline Country & Key Biodiversity Area & Species scientific name & $\begin{array}{c}\text { IUCN Red List } \\
\text { Category }\end{array}$ \\
\hline & & Zanthoxylum flavum & VU \\
\hline & Vieques & Calyptranthes thomasiana & EN \\
\hline & & Chrysophyllum pauciflorum & VU \\
\hline & & Erythrina eggersii & EN \\
\hline & & Goetzea elegans & EN \\
\hline & & Guaiacum officinale & EN \\
\hline & & Manilkara pleeana & VU \\
\hline & & Maytenus cymosa & EN \\
\hline & & Stahlia monosperma & EN \\
\hline & & Stenoderma rufum & VU \\
\hline \multirow[t]{2}{*}{ Saba } & Saba & Alsophis rufiventris & EN \\
\hline & & Guaiacum officinale & EN \\
\hline \multirow[t]{6}{*}{ St Barthelemy } & Colombier & Alsophis rijersmai & EN \\
\hline & & Iguana delicatissima & VU \\
\hline & Gustavio to Anse Toiny & Alsophis rijersmai & EN \\
\hline & & Iguana delicatissima & VU \\
\hline & Ile Fourchue and satellites & Iguana delicatissima & VU \\
\hline & Petite Islette & Iguana delicatissima & VU \\
\hline \multirow[t]{4}{*}{ St Eustatius } & Boven & Alsophis rufiventris & EN \\
\hline & & Iguana delicatissima & VU \\
\hline & The Quill & Alsophis rufiventris & EN \\
\hline & & Iguana delicatissima & VU \\
\hline St Kitts and Nevis & Cayon to Key & Dermochelys coriacea & $\mathrm{CR}$ \\
\hline \multirow[t]{16}{*}{ St Lucia } & Government Forest Reserve & Amazona versicolor & VU \\
\hline & & Leucopeza semperi & $\mathrm{CR}$ \\
\hline & & Melanospiza richardsoni & EN \\
\hline & & Swietenia mahagoni & EN \\
\hline & & Turdus Iherminieri & VU \\
\hline & Mandele Dry Forest & Amazona versicolor & VU \\
\hline & & Cnemidophorus vanzoi & VU \\
\hline & & Melanospiza richardsoni & EN \\
\hline & & Ramphocinclus brachyurus & EN \\
\hline & North-east coast & Melanospiza richardsoni & EN \\
\hline & & Ramphocinclus brachyurus & EN \\
\hline & Pitons Management Area & Melanospiza richardsoni & EN \\
\hline & Point Sables & Cnemidophorus vanzoi & VU \\
\hline & & Liophis ornatus & EN \\
\hline & & Melanospiza richardsoni & EN \\
\hline & Rat Island & Cnemidophorus vanzoi & VU \\
\hline St Martin & Tintamarre & Iguana delicatissima & VU \\
\hline \multirow[t]{15}{*}{$\begin{array}{l}\text { St Vincent and the } \\
\text { Grenadines }\end{array}$} & Colonarie Forest Reserve & Amazona guildingii & VU \\
\hline & & Catharopeza bishopi & EN \\
\hline & & Chironius vincenti & $\mathrm{CR}$ \\
\hline & & Pristimantis shrevei & EN \\
\hline & Cumberland Forest Reserve & Amazona guildingii & VU \\
\hline & & Catharopeza bishopi & EN \\
\hline & & Chironius vincenti & $\mathrm{CR}$ \\
\hline & & Pristimantis shrevei & EN \\
\hline & Dalaway Forest Reserve & Amazona guildingii & VU \\
\hline & & Catharopeza bishopi & EN \\
\hline & & Chironius vincenti & $\mathrm{CR}$ \\
\hline & & Pristimantis shrevei & EN \\
\hline & Kingstown Forest Reserve & Amazona guildingii & VU \\
\hline & & Catharopeza bishopi & EN \\
\hline & & Chironius vincenti & $\mathrm{CR}$ \\
\hline
\end{tabular}




\begin{tabular}{|c|c|c|c|}
\hline Country & Key Biodiversity Area & Species scientific name & $\begin{array}{l}\text { IUCN Red List } \\
\text { Category }\end{array}$ \\
\hline & & Pristimantis shrevei & EN \\
\hline & La Soufrière National Park & Amazona guildingii & VU \\
\hline & & Catharopeza bishopi & EN \\
\hline & & Chironius vincenti & CR \\
\hline & & Pristimantis shrevei & EN \\
\hline & Mount Pleasant Forest Reserve & Amazona guildingii & VU \\
\hline & & Catharopeza bishopi & EN \\
\hline & & Chironius vincenti & $\mathrm{CR}$ \\
\hline & & Pristimantis shrevei & EN \\
\hline & Richmond Forest Reserve & Amazona guildingii & VU \\
\hline & & Catharopeza bishopi & EN \\
\hline & & Chironius vincenti & $\mathrm{CR}$ \\
\hline & & Pristimantis shrevei & EN \\
\hline \multirow[t]{13}{*}{ Turks and Caicos Islands } & Caicos Bank Southern Cays & Cyclura carinata & $\mathrm{CR}$ \\
\hline & Chalk Sound & Cyclura carinata & $\mathrm{CR}$ \\
\hline & East Bay Islands & Cyclura carinata & $\mathrm{CR}$ \\
\hline & East Caicos and adjacent areas & Cyclura carinata & $\mathrm{CR}$ \\
\hline & & Dendrocygna arborea & VU \\
\hline & $\begin{array}{l}\text { Fish Ponds and Crossing Place Trail, Middle } \\
\text { Caicos }\end{array}$ & Dendrocygna arborea & VU \\
\hline & Middle Caicos Forest & Dendrocygna arborea & VU \\
\hline & North, Middle and East Caicos Ramsar Site & Cyclura carinata & $\mathrm{CR}$ \\
\hline & & Dendrocygna arborea & VU \\
\hline & & Swietenia mahagoni & EN \\
\hline & Princess Alexandra Land and Sea & Cyclura carinata & CR \\
\hline & Salt Cay Creek and Salinas & Cyclura carinata & $\mathrm{CR}$ \\
\hline & Turks Bank Seabird Cays & Cyclura carinata & $\mathrm{CR}$ \\
\hline \multirow[t]{29}{*}{ US Virgin Islands } & Buck Island & Ameiva polops & CR \\
\hline & & Chelonia mydas & EN \\
\hline & & Eretmochelys imbricata & $\mathrm{CR}$ \\
\hline & East End Bay & Chelonia mydas & EN \\
\hline & Hassel Island & Eleutherodactylus lentus & EN \\
\hline & Jack's and Issac's Bays & Chelonia mydas & EN \\
\hline & John Brewer's Bay & Eleutherodactylus lentus & EN \\
\hline & Magens Bay & Chrysophyllum pauciflorum & VU \\
\hline & & Eleutherodactylus lentus & EN \\
\hline & & Erythrina eggersii & EN \\
\hline & & Zanthoxylum thomasianum & EN \\
\hline & Northwestern St Croix & Eleutherodactylus lentus & EN \\
\hline & & Stenoderma rufum & $\mathrm{VU}$ \\
\hline & Protestant Cay & Ameiva polops & $\mathrm{CR}$ \\
\hline & Ruth Cay & Ameiva polops & $\mathrm{CR}$ \\
\hline & Sandy Point National Wildlife & Chelonia mydas & EN \\
\hline & & Dermochelys coriacea & $\mathrm{CR}$ \\
\hline & & Eretmochelys imbricata & $\mathrm{CR}$ \\
\hline & Southgate and Green Cay & Ameiva polops & $\mathrm{CR}$ \\
\hline & St John & Calyptranthes thomasiana & EN \\
\hline & & Chrysophyllum pauciflorum & VU \\
\hline & & Eleutherodactylus lentus & EN \\
\hline & & Eleutherodactylus schwartzi & EN \\
\hline & & Erythrina eggersii & EN \\
\hline & & Guaiacum officinale & EN \\
\hline & & Machaonia woodburyana & $\mathrm{CR}$ \\
\hline & & Maytenus cymosa & EN \\
\hline & & Stenoderma rufum & $\mathrm{VU}$ \\
\hline & & Zanthoxylum thomasianum & EN \\
\hline
\end{tabular}


Spanish Abstract: Resumen: El hotspot de las islas del Caribe es excepcionalmente importante para la conservación de la biodiversidad global por sus altos niveles de endemismo y amenaza. Un total de 755 especies de plantas y vertebrados del Caribe son consideradas globalmente amenazadas, colocándolo en uno de los más altos hotspot de biodiversidad en términos de nivel de amenaza. En el 2009, las Áreas Claves para la Biodiversidad (KBAs) fueron identificadas para las islas del Caribe a través de un análisis de data y literatura accesible a nivel regional, seguido por consultas extensas a expertos a nivel nacional. Aplicando el criterio de la Vulnerabilidad, un total de 284 Áreas Claves para la Biodiversidad fueron definidas y delimitadas conteniendo 409 (54\%) de las especies amenazadas de la región. De éstas, 144 (o 51\%) solapan parcialmente o completamente con áreas protegidas. Cockpit Country, seguida por Litchfield Mountain - Matheson's Run, Blue Mountains (todos en Jamaica) y Massif de la Hotte (Haití) fueron encontrados de sostener números excepcionalmente altos de taxones globalmente amenazados, con más de 40 de estas especies en cada sitio. Las Áreas Claves para la Biodiversidad, basadas en las Áreas Importantes para la Conservación de las Aves, proporcionan un marco valioso contra el cual revisar la adecuación de sistemas de áreas protegidas nacionales existentes y también priorizar cuales especies y sitios requieren la atención más urgente para su conservación.

Acknowledgements: The definition of Key Biodiversity Areas in the Caribbean Islands Biodiversity Hotspot was the result of generous feedback and assistance from a wide range of people and institutions. We would especially like to thank the coordinators for facilitating workshops and national processes in The Bahamas (Bahamas National Trust), Dominican Republic (Grupo Jaragua), Haiti (Jean Vilmond Hilaire) and Puerto Rico (Mayra Vincenty and Verónica Mendez Gallardo). A regional consultation group was also instrumental in facilitating a wide review of the priorities, and comprised the following individuals: Philippe Bayard, Judi Clarke, Andrew Dobson, Julia Horrocks, Sixto Inchaustegui, Brad Keitt, Penny Langhammer, Nicole Leotaud, Sarah McIntosh, Kalli de Meyer, Sarah Sanders, Lisa Sorenson, Helene Souan, Jack Tordoff, Alessandra Vazella-Khouri, Doug Ryan, Hannah Stevens, Ian May, James Millet, Mark O'Connell, Matthew Foster, Michele Zador, Nigel Varty, Gill Bunting and Joe Wunderle. The process was also assisted by the following individual experts: Abdel Abellard, Sandra Buckner, Sean Carrington, Colin Clubbe, Rhon Connor, Mat Cottam, William Crosse, Jenny Daltry, Liliana Davalos, Alison Duncan, Paul Edgar, Philippe Feldmann, Tony Gent, Martin Hamilton, Hugh Genoways, Gerard Gray, Blair Hedges, Geoff Hilton, Arlington James, Kimberly John, Charles Knapp, Gary Kwiecinski, Vincent Lemoine, Anthony Levesque, Don McFarlane, Matthew Morton, Farah Mukhida, Andreas Oberli, Scott Pedersen, Laura Perdomo, Tineke Prins, Bonnie Rusk, Ronald Stefan Stewart, Ann Sutton, Armando Rodríguez and Joel Timyan. Lastly, individuals from the following institutions provided invaluable information and feedback before, during and after the various workshops: Adventours; AEVA; AMAZONA; American Bird Conservancy; Amigos de Sian Ka'an A.C.; Anguilla National Trust; Arizona State University; Asa Wright Nature Centre; Avian Eyes Birding Group; Bahamas Ministry of Tourism and Aviation; Bahamas National Trust; Bahamas Outdoors Limited; Bat Conservation International; Bermuda Audubon Society; Bonaire Parrot project, University of Sheffield; Boston University; BVI National Parks Trust; CARE ; Caribbean Coastal Area Management; Caribbean Natural Resources Institute; Centre d'Information Geospatiale; Centro de Aprendizaje para la Conservación de Sarapiquí; Centro para la Conservación y Ecodesarrollo de la Bahía de Samaná; The Claremont Colleges; Columbia University; CONHAME; Consorcio Ambiental Dominicano; Consultora Hernández; Cornell Laboratory of Ornithology; Department of Conservation Sciences, Bermuda; Department of Natural Resources and the Environment,
Puerto Rico; Department of the Environment Anguilla; Department Environment, Montserrat Gov : Durham University; Durrell Wildlife Conservation Trust; Dutch Caribbean Nature Alliance; Econcerns Ltd; ENAF; Environment for the Americas; Environmental Awareness Group of Antigua and Barbuda; Environmental Management Division, Office of the Prime Minister (Jamaica); Environmental Protection in the Caribbean; Faculté d'Agronomie et de Medicine Veterinaire; Fauna and Flora International; Fenad; Fermata Inc; Fondation EcosOphique; Fondation Macaya; Fondation Seguin; Forestry Department; Forestry, Wildlife and Parks Division- Dominica; Fundación para el Desarrollo Humano-PROGRESSIO; Grand Bahama Nature Tours; Grupo Jaragua, Inc. Herpetological Conservation Trust; Institute of Jamaica, Natural History Museum of Jamaica; Institute of Marine Affairs; Instituto Tecnológico de Santo Domingo; Island Conservation; IUCN Iguana Specialist Group; Jadora International LLC; Jamaican Caves Organisation; Jamaica Forestry Department; Jardín Botánico Nacional John D. and Catherine T. MacArthur Foundation Klamath Bird Observatory; Laboratorio UASDSteven; MDE; Ministry of Energy and Mining Ministry of Agriculture, Montserrat; Museo Nacional de Historia Natural; National Audubon Society; National Environment and Planning Agency; National Trust for the Cayman Islands Natouraves; Negril Area Environmental Protection Trust; New York Botanical Garden; Northern Jamaica Conservation Association OJJUOES; ONCFS National Hunting and Wildlife Agency; Optics for the Tropics; Pacific Union College; Panos Caribbean; Parque Zoológico Nacional; Planning Institute of Jamaica; Plan Conservation Centre; Platte River Whooping Crane Maintenance Trust; Population Media
Center; Programa Naciones Unidas para el Desarrollo; Puerto Rico Department of Natural Resources; PWD Gun Club; Rare; REPIE; Ross University School of Medicine, Department of Biochemistry; Royal Society for the Protection of Birds; Secretaría de Estado de Educación Superior Ciencia y Tecnología/UASD; Secretaría de Estado de Medio Ambiente; Sociedad Ornitológica Hispaniola: Sociedad Ornitológica Puertoriqueña; Société Audubon Haïti; Société Financiere de Developpement ; Society for the Conservation and Study of Caribbean Birds; South Dakota State University; State University of New York at Stony Brook; Stichting Nationale Parken Bonaire; Subsecretaría de Planificación y Desarrollo; Sustainable Grenadines Project; The Nature Conservancy; The Nature Conservancy-DR; The Nature Conservancy, USVI; Trelawny Gun Club; Tourism Product Development Co Ltd; UCH/DES; UK Overseas Territories, Conservation Forum; UNDP; UNEPCaribbean Environment Program; Universidad Autónoma de Santo Domingo; Universidad de Cornell; Université d'Etat d'Haiti; Université Quisqueya; University of California; University of Nebraska State Museum; University of New Brunswick; University of Pittsburgh, National Aviary; University of Puerto Rico; University of Scranton; University of Simon Bolivar; University of the West Indies; University of the West Indies - Cave Hill and Mona Campuses; University of the West Indies, Life Sciences; U.S. Agency for International Development / DAl; USDA Forest Service, Int'I Institute of Tropical Forestry; U.S. Fish and Wildlife Service; U.S. Fish and Wildlife Service, Div. Scientific Authority; U.S. Fish and Wildlife Service, Latin America and Caribbean Region; U.S. Forest Service, Wings Across the Americas; USFS International Institute for Tropical Forestry; U.S. Geological Survey; USVI 
Division of Fish and Wildlife; Vermont Center for Ecostudies; WIDECAST; Windsor Research Centre; Zoological Museum of Amsterdam; Zoológico Nacional/Universidad Autónoma de Santo Domingo; Zoological Society of San Diego; Zoological Society of Trinidad and Tobago. We would like to thank the Critical Ecosystem Partnership Fund for the financial and technical support. The Critical Ecosystem Partnership Fund is a joint initiative of l'Agence Française de Développement, Conservation International, the Global Environmental Facility, the Government of Japan, the MacArthur Foundation and the World Bank. A fundamental goal of the fund is to ensure civil society is engaged in biodiversity conservation.

\section{Author Details and Contribution:}

VERÓNICA ANADÓN-IRIZARRY is the Caribbean Program Manager for BirdLife International. She has been supporting the development, management and implementation of BirdLife's Caribbean Program since 2005. She reviewed boundaries, processed, analyzed and confirmed data for all the threatened species contained in the 284 Caribbean KBAs included in the Caribbean Hotspot Ecosystem Profile. She is lead author of this manuscript.

DAVID C. WEGE is BirdLife International's Senior Caribbean Program Manager. He has led the development of a comprehensive program of Caribbean conservation - delivered by BirdLife's network of national partners. He led the Critical Ecosystem Partnership Fund project to develop the Caribbean Hotspot Ecosystem Profile, defining KBAs that provide the scientific basis for CEPF's hotspot investment strategy. He provided the lead author with editorial guidance, technical support and advice based on our joint work to define the hotspot's KBAs.

Amy Upgren is the Advisor, Conservation Priorities, with the Conservation Priorities and Outreach team at Conservation International (CI). In conjunction with $\mathrm{Cl}$ regional offices and partners, she identifies priorities to safeguard biodiversity and human well-being. She is currently working to develop Cl's institutional framework for identifying geographic priorities and to link science staff in $\mathrm{Cl}$ headquarters with technical staff in the field. She is a member of the metrics and priority setting team and the freshwater and species teams. She compiled data, supported analysis, KBA delineation and prioritization, and edited the manuscript.

RICHARD Young is Head of Conservation Science at the Durrell Wildlife Conservation Trust, and leads research programmes to support the design, management and evaluation of Durrell's conservation efforts in Madagascar, Mauritius, the Caribbean, the Pacific and India. $\mathrm{He}$ is Co-Chair of the IUCN/SSC Small Mammal Specialist Group and a Visiting Lecturer at the University of Bath, UK. He compiled publicly available spatial data on the distributions of globally threatened amphibians and reptiles for the Caribbean, including liaising with global experts to gather unpublished data, and drafted KBAs boundaries for review by national committees. He provided comments on the design of the paper and edited two drafts of the manuscript.

BRIAN Boom serves as Director of the Caribbean Biodiversity Program and Bassett Maguire Curator of Botany at The New York Botanical Garden. In collaboration with Cuban scientists, his current principal focus is to identify and assess Cuba's most vulnerable plant species in the face of climate change and habitat loss. For this study and paper, he compiled spatial distribution data on globally threatened plan species in the Caribbean region, and, with NYBG colleague Hannah Stevens, mapped these occurrences to propose new KBAs.

YolANDA M. LEÓN is a research professor a Instituto Tecnológico de Santo Domingo managing the Geographic Information Systems Laboratory and specializing in environmental applications of GIS. She is the President of Grupo Jaragua, whose mission is to preserve the biodiversity of Hispaniola (with emphasis on the Jaragua-Bahoruco-Enriquillo UNESCO Biosphere Reserve) through work with loca communities, environmental advocacy and education. She co-coordinated the Dominican Republic's KBA stakeholder workshop, assisted with KBA identification, processed experts information, developed shapefiles and edited the manuscript.

YVONNE ARIAS is the Executive Director and founder member of Grupo Jaragua and Vice-president of the IUCN Committee for the Dominican Republic. She was recently recognized with the Woman Merit Medal in the category of Science and Technology for the Conservation of the Environment and the Natura Resources granted by the Dominican Republic government. She has 30 years of experience in ecology and herpetology, protected areas and environmental education. She is the coordinato and co-author of the Important Bird Areas and coordinated the Dominican Republic's KBA stakeholder workshop, and edited the manuscript.

Kellee Koenig is the GIS Manager and Cartographer at Conservation International. She contributed to the study by compiling and helping correct the GIS data.

Alcides L. MoRales is a biologist working on wetland enhancement and terrestrial bird surveys, and President of Sociedad Ornitológica Puertorriqueña, Inc. $\mathrm{He}$ is experienced in wildlife research and conservation, working and collaborating with governmental agencies, universities, non-governmental organizations as a scientific research assistant and advise both with plants and animals (especially birds) He contributed field observations, report and data to the 27 Puerto Rican KBAs, and provided general review to the manuscript.

WAYNE BURKE is Barbados Project Manager of BirdLife International currently working on a Neotropical Migratory Bird Conservation Act funded project focusing on increasing survival prospects for Neotropical migratory shorebirds on Barbados. He acted as a facilitator and resource for species information and site descriptions for Barbados and the Lesser Antilles, and provided general review to the manuscript.

Amiro PÉREZ-Leroux is the Interim Director for the Americas Division of BirdLife International. He is in charge of overseeing the implementation of key programmes and projects together with project managers, annual workplans, budget review, and financial issues. He coordinates and oversees the implementation of major fundraising initiatives with key donors including the financial and technical reporting. He lead the design and facilitation of three national and one regional workshops that were the basis for the CEPF Caribbean profile and this research. CATHERINE LeVY is an independent researcher and co-represents Windsor Research Centre on the Steering Committee of the UNDP project "Strengthening the operational and financial sustainability of the National Protected Area System". She has previously been President of BirdLife Jamaica and the Society for the Conservation and Study of Caribbean Birds. For over 10 years she has been on the Management Board of the Natural History Museum, and a Director of the Windsor Research Centre. She was the co-author of Jamaica's Important Bird Areas, and provided general review to this manuscript.

SUSAN KOENIG holds a doctorate from the School of Forestry and Environmental Studies, Yale University. She is a founding director of Windsor Research Centre, an environmental NGO located $5 \mathrm{~km}$ inside Jamaica's Cockpit Country and which has a mission to conserve Cockpit Country through a programme of research, education outreach, and advocacy. She is co-author of the Jamaica section in the publication Important Bird Areas of the Caribbean. She collated the amphibian, reptile, and bat datasets, which were utilized by Jamaica's National Ecological Gap Assessment Report and which subsequently were provided to this study. She participated in Jamaica's national workshop, which defined the island's KBAs, and assisted with editorial review of the manuscript.

LYNN GAPE is the Deputy Executive Director of the Bahamas National Trust (BNT). She was recently recognized for her 20 years of service to the BNT. She serves on the National Biodiversity Sub Committee and acts as an advisor and sometimes instructor in the Bahamas Ministry of Tourism Tour Guide Training Programme. She has a keen personal interest in birding and was one of the founding members of the BNT's Ornithology group. She acted as a resource for species information and site descriptions with regards to the 26 Bahamas KBA's.

Predensa Moore is the Science Research Officer for The Bahamas National Trust. She is the coordinator for the International Piping Plover Census in The Bahamas and author of The Bahamas section in the Important Bird Areas for the Caribbean directory. She reviewed the species information for The Bahamas KBAs. 